\title{
Impact of current treatments on liver disease, glucose metabolism and cardiovascular risk in non-alcoholic fatty liver disease (NAFLD): a systematic review and meta-analysis of randomised trials
}

\author{
G. Musso • M. Cassader • F. Rosina • R. Gambino
}

Received: 2 June 2011 / Accepted: 2 December 2011 / Published online: 27 January 2012

(C) Springer-Verlag 2012

\begin{abstract}
Aims/hypothesis Non-alcoholic fatty liver disease (NAFLD) encompasses a spectrum ranging from simple steatosis to non-alcoholic steatohepatitis (NASH): NAFLD causes an increased risk of cardiovascular disease, diabetes and liverrelated complications (the latter confined to NASH). The effect of proposed treatments on liver disease, glucose metabolism and cardiovascular risk in NAFLD is unknown. We reviewed the evidence for the management of liver disease and cardiometabolic risk in NAFLD.

Methods Publications through November 2011 were systematically reviewed by two authors. Outcomes evaluated though standard methods were: histological/radiological/biochemical features of NAFLD, variables of glucose metabolism and cardiovascular risk factors. Seventy-eight randomised trials were included (38 in NASH, 40 in NAFLD): $41 \%$ assessed post-treatment histology, $71 \%$ assessed glucose metabolism and $88 \%$ assessed cardiovascular risk factors. Lifestyle intervention, thiazolidinediones, metformin and antioxidants were most extensively evaluated.

Results Lifestyle-induced weight loss was safe and improved cardio-metabolic risk profile; a weight loss $\geq 7 \%$ improved histological disease activity, but was achieved by $<50 \%$
\end{abstract}

Electronic supplementary material The online version of this article (doi:10.1007/s00125-011-2446-4) contains peer-reviewed but unedited supplementary material, which is available to authorised users.

G. Musso $(\bowtie) \cdot$ F. Rosina

Gradenigo Hospital,

C.so Regina Margherita 8,

10132 Turin, Italy

e-mail: giovanni_musso@yahoo.it

M. Cassader $\cdot$ R. Gambino

Department of Internal Medicine, University of Turin,

Turin, Italy patients. Statins and polyunsaturated fatty acids improved steatosis, but their effects on liver histology are unknown. Thiazolidinediones improved histological disease activity, glucose, lipid and inflammatory variables and delayed fibrosis progression. Pioglitazone also improved blood pressure. Weight gain (up to 4.8\%) was common. Antioxidants yielded mixed histological results: vitamin $\mathrm{E}$ improved histological disease activity when administered for 2 years, but increased insulin resistance and plasma triacylglycerols.

Conclusions/interpretation Weight loss is safe, and improves liver histology and cardio-metabolic profile. For patients not responding to lifestyle intervention, pioglitazone improves histological disease activity, slows fibrosis progression and extensively ameliorates cardio-metabolic endpoints. Further randomised controlled trials (RCTs) of adequate size and duration will assess long-term safety and efficacy of proposed treatments on clinical outcomes.

Keywords Fatty liver Human - Management . Meta-analysis $\cdot$ NAFLD $\cdot$ NASH $\cdot$ Systematic review

$\begin{array}{ll}\text { Abbreviations } \\ \text { ALT } & \text { Alanine aminotransferase } \\ \text { CB1 } & \text { Cannabinoid type 1 receptor } \\ \text { CRP } & \text { C-reactive protein } \\ \text { CT } & \text { Computed tomography } \\ \text { CVD } & \text { Cardiovascular disease } \\ \text { EOT } & \text { End of treatment } \\ \text { FPG } & \text { Fasting plasma glucose } \\ \text { FLIRT } & \text { Fatty Liver Improvement with Rosiglitazone } \\ & \text { Therapy } \\ \text { FXR } & \text { Farnesoid X receptor } \\ \text { GLP-1 } & \text { Glucagon-like peptide-1 } \\ \text { GREACE } & \text { Greek Atorvastatin and Coronary Heart } \\ & \text { Disease Evaluation }\end{array}$




$\begin{array}{ll}\text { MRS } & \text { Magnetic resonance spectroscopy } \\ \text { NAFLD } & \text { Non-alcoholic fatty liver disease } \\ \text { NAS } & \text { NAFLD activity score } \\ \text { NASH } & \text { Non-alcoholic steatohepatitis } \\ \text { NMR } & \begin{array}{l}\text { Nuclear magnetic resonance } \\ \text { PGC1 } \alpha\end{array} \\ & \begin{array}{l}\text { Peroxisome proliferator activated receptor- } \gamma \\ \text { coactivator } 1 \alpha\end{array} \\ \text { PIVENS } & \begin{array}{l}\text { Pioglitazone Versus Vitamin E Versus Placebo } \\ \text { for the Treatment of Non-diabetic Patients with }\end{array} \\ & \text { NASH } \\ \text { PPAR } & \text { Peroxisome proliferator activated receptor } \\ \text { PUFA } & \begin{array}{l}\text { Polyunsaturated fatty acid } \\ \text { RCT }\end{array} \\ \text { Randomised controlled trial } \\ \text { TG } & \text { Triacylglycerol } \\ \text { UDCA } & \text { Ursodeoxycholic acid } \\ \text { WMD } & \text { Weighed mean difference }\end{array}$

\section{Introduction}

Non-alcoholic fatty liver disease (NAFLD) affects $30 \%$ of the general adult population and $60-80 \%$ of diabetic and obese patients $[1,2]$. NAFLD encompasses a histological spectrum ranging from simple steatosis (SS) to steatosis plus necroinflammation (non-alcoholic steatohepatitis, NASH), with or without fibrosis, that can only be differentiated by liver biopsy. NAFLD carries an increased risk of (1) liver-related complications: whereas SS is considered to have a benign hepatological prognosis, NASH progresses to cirrhosis in $20-25 \%$ of cases over 10 years [1]; (2) cardio-metabolic complications: NAFLD confers an increased risk of cardiovascular disease (CVD) and diabetes [3] both directly and through its association with other cardio-metabolic abnormalities, including obesity and metabolic syndrome [4]. Therefore, the impact of proposed treatments on cardio-metabolic profile, as well as on liver disease, should be evaluated. We systematically reviewed the effect of current non-surgical treatments on liver disease and cardio-metabolic risk in NAFLD.

\section{Methods}

Data sources and study selection

A detailed description of data sources and searches, and of study selection, is reported in the electronic supplementary material (ESM).

Outcome measures

Liver disease Primary outcome measures were incident cirrhosis/liver failure/hepatocellular carcinoma and improvement in hepatic histological features (steatosis, hepatocellular ballooning, lobular inflammation, fibrosis and, when separate histological features were unavailable, NAFLD activity score, NAS, which is the sum of steatosis, hepatocellular ballooning and lobular inflammation); wherever possible, the impact on fibrosis progression (i.e. the number of patients with unchanged or improved fibrosis stage) was also assessed. When these outcomes were unavailable, changes in radiological steatosis (by ultrasonography, nuclear magnetic resonance [NMR] magnetic resonance spectroscopy [MRS] or computed tomography $[\mathrm{CT}]$ ), and in serum alanine aminotransferase (ALT) were evaluated.

Glucose metabolism We evaluated incident diabetes, fasting plasma glucose (FPG), glucose tolerance (as assessed by a standard OGTT), $\mathrm{HbA}_{1 \mathrm{c}}$, HOMA index and other variables related to insulin sensitivity (hepatic and extrahepatic) and insulin secretion, BMI and abdominal obesity (assessed by anthropometry or by NMR/CT).

Cardiovascular risk We evaluated incident cardiovascular events, BP, plasma lipids (triacylglycerol, LDL- and HDLcholesterol) and inflammatory markers/cytokines, including C-reactive protein (CRP), adiponectin, interleukin-6 and TNF- $\alpha$.

Incident adverse events were also evaluated.

The quality of randomised controlled trials (RCTs) was assessed by the Cochrane Risk of Bias Tool (score range: $0-8$ ) [5]. RCTs scoring $>6$ were arbitrarily considered as having a low bias risk.

\section{Results}

The agreement for study selection between the two reviewers was good ( $\mathrm{K}$ coefficient $=0.86$ ). We retrieved 78 RCTs (47 with a low risk of bias), variably reporting posttreatment changes in liver-related, glucose and cardiovascular variables (Table 1; ESM Fig. 1; ESM Tables 1-5).

\section{Weight loss}

Eight RCTs (373 participants, 39\% diabetic; six RCTs with a low risk of bias, four RCTs with post-treatment histology) assessed the effect of lifestyle- or drug-induced weight loss in NAFLD [6-13] (ESM Table 1).

Liver disease Although a $\geq 5 \%$ weight loss improved hepatic steatosis, a $\geq 7 \%$ weight loss also improved NAS (Fig. 1); fibrosis was unchanged (not shown). The threshold of $7 \%$ weight loss was achieved by $<50 \%$ of patients, even with intensive multidisciplinary lifestyle intervention $[8,10]$. Two 
Table 1 Items related to liver disease, glucose metabolism and cardiovascular risk and the percentage of RCTs assessing their post-treatment changes (total: 78 RCTs included)
FGF, fibroblast growth factor; FSIVGTT, frequently sampled intravenous glucose tolerance test; ICAM, intercellular adhesion molecule; TGF, transforming growth factor; VCAM, vascular cellular adhesion molecule
Item assessed

Method

RCTs with posttreatment changes $(\%)$

\section{Liver disease \\ Liver histology \\ Radiological steatosis}

Liver enzymes

Adiposity

Whole body adiposity

Abdominal adiposity

Glucose homeostasis

Pancreatic beta cell function

Insulin sensitivity

Plasma glucose control

FPG

Glucose tolerance

$\mathrm{HbA}_{1 \mathrm{c}}$

Plasma lipids

Fasting plasma triacylglycerols, total cholesterol/LDL-cholesterol/ HDL-cholesterol

BP

Systolic/diastolic BP

Chronic systemic inflammation

Pro-/anti-inflammatory cytokines

$\begin{array}{ll}\text { Liver biopsy } & 41 \\ & 45 \\ \text { Ultrasound } & 17 \\ \text { MRI } & 21 \\ \text { CT } & 8 \\ \text { AST, ALT, GGT } & 93\end{array}$

BMI 99

37

Waist 24

Waist-on-hip ratio 4

MRI 9

CT 4

OGTT-derived indices of 3 pancreatic beta cell function

Fasting indices (HOMA, QUICKI) 55

OGTT-derived indices 8

FSIVGTT 1

Hyperinsulinemic euglycaemic 9 glucose clamp-derived indices

76

$2 \mathrm{~h}$ plasma glucose on OGTT 17

$-$
Adiponectin 24

C-reactive protein 19

TNF- $\alpha \quad 8$

Interleukin-6 4

TGF- $\beta$, FGF-18, ICAM-1, VCAM-1 2
RCTs suggested no additional NAS improvement with $>10 \%$ weight loss, but the existence of a lower and an upper threshold weight loss for improving histological disease activity needs further confirmation (Fig. 2).

There was no significant publication bias (ESM Fig. 2).

Glucose metabolism and cardiovascular risk Weight loss substantially improved HOMA, FPG, glucose tolerance and plasma lipids (ESM Table 1). Two RCTs also showed an improvement in plasma adiponectin [8, 12]. Among drugs inducing weight loss, orlistat was safe, well-tolerated with minor adverse gastrointestinal complaints not requiring discontinuation of therapy, but conferred no additional cardio-metabolic or histological benefit over lifestyle intervention alone [7, 12]. There was no significant publication bias for assessed outcomes (not reported). 


\begin{tabular}{lll|l}
\hline & $\begin{array}{c}\text { Mean difference } \\
\text { IV, fixed, 95\% Cl }\end{array}$ \\
Authors [ref] & $-2.50(-3.52,-1.48)$ \\
\hline Harrison et al, 2009 [12] & $-1.10(-2.11,-0.09)$ \\
Nobili et al, 2008 [11] & $-2.30(-3.38,-1.22)$ \\
Promrat et al, 2009 [10] & Mean difference \\
Vilar Gomez et al, 2009 [98] & $-2.40(-3.51,-1.29)$ & \\
Total (95\% Cl) & $-2.05(-2.58,-1.53)$ &
\end{tabular}

Fig. 1 Forest plot of RCTs comparing the effect of different degrees of weight loss (\%) on histological NAS. Outcome: mean differences in NAS following weight loss $\geq 7 \%$ vs weight loss $<7 \%$. IV, inverse variance

Long-term durability of achieved benefits and safety of weight loss are unknown.

Physical exercise alone

Reduced aerobic exercise has been linked to the presence and severity of cardio-metabolic and liver disease in NAFLD through several potential mechanisms: reduced
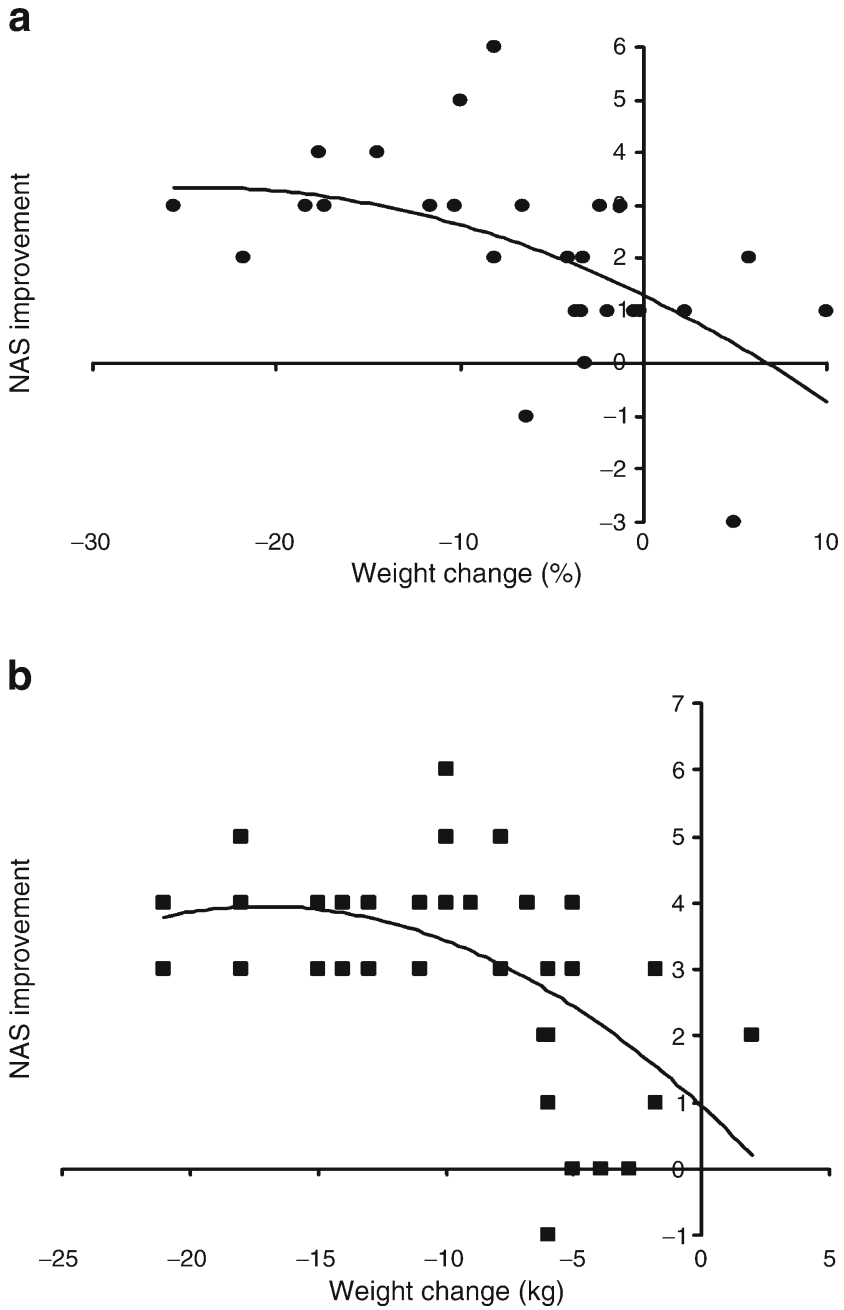

Fig. 2 Impact of different degrees of weight loss on histological NAS in two RCTs (adapted from (a) Promrat et al [10] and (b) Vilar Gomez et al [98]) hepatic and muscle adenosine monophosphate-activated protein kinase (AMPK)-mediated NEFA oxidation, increased postprandial hepatic lipogenesis, visceral fat-derived NEFA and proinflammatory adipokine overflow to the liver [14-17].

Five RCTs (four RCTs with a low risk of bias) evaluated the effects of 3-6 months of moderate-intensity aerobic exercise alone in NAFLD [13, 18-21] (ESM Table 1).

Liver disease Exercise improved MRS-assessed steatosis and ALT levels (Fig. 3). In the only RCT with posttreatment histology, NAS was unchanged [13]. There was no significant publication bias (ESM Fig. 2)

Glucose metabolism and cardiovascular risk Despite no significant body weight changes, exercise improved waist circumference, HOMA, FPG, $\mathrm{HbA}_{1 \mathrm{c}}$, LDL-cholesterol and triacylglycerol (TG) (Fig. 3). One RCT reported no effect of physical exercise on HDL-cholesterol [20]. No data on inflammatory markers/adipokines are available. There was no significant publication bias for assessed outcomes (not reported).

An analysis of the reasons for dropping out of exercisebased treatments found that NAFLD patients understand the benefits of exercise but lack confidence to perform it, and are afraid of falling, suggesting that these modifiable factors should be targeted to improve compliance to exercise of these patients [22].

\section{Dietary composition manipulation}

The optimal nutrient dietary composition for NAFLD is unknown. Three RCTs compared the effect of lowcarbohydrate versus low-fat caloric restriction [23-25] (ESM Table 1).

Liver disease The two regimens yielded similar liver fat and ALT reduction (Fig. 4).

Glucose metabolism and cardiovascular risk The two regimens yielded similar weight loss and improved HOMA, pancreatic beta cell function [24], TG, blood pressure [25], CRP [24] and adiponectin to a similar extent (Fig. 4). For TG and HOMA heterogeneity was high, being explained by the different baseline features of study populations: low-carbohydrate diet significantly improved plasma TG and HOMA index when hypertriacylglycerolaemic [25] or glucose-intolerant [23] NAFLD patients, respectively, were enrolled. Furthermore, in glucose-intolerant NAFLD individuals, low-carbohydrate caloric restriction significantly improved hepatic insulin sensitivity compared with low-fat diet [23].

Low-carbohydrate diet significantly reduced waist circumference and FPG compared with low-fat diet, which in 
a

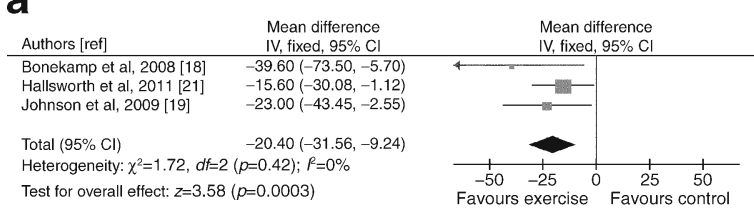

C

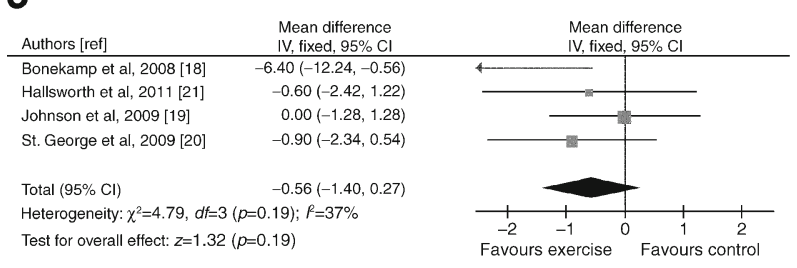

e

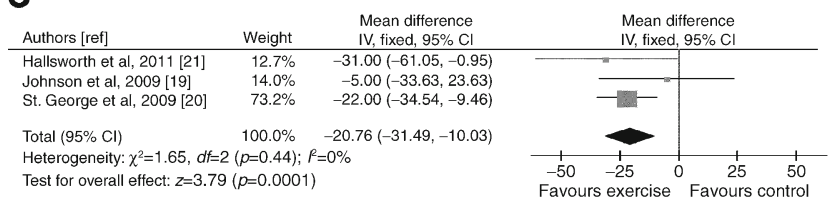

9

Authors [ref] Favours exercise Favours control

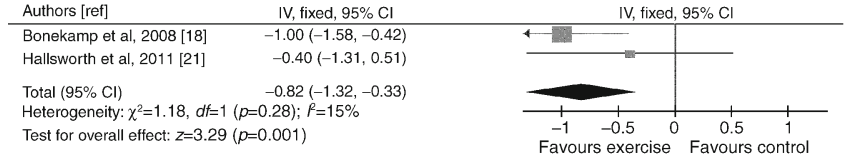

b

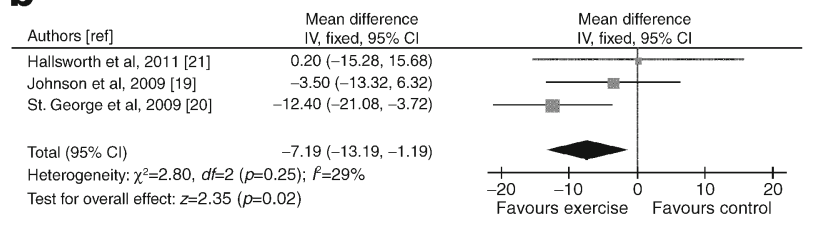

d

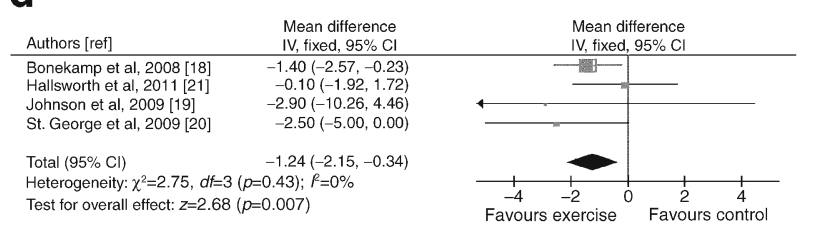

f

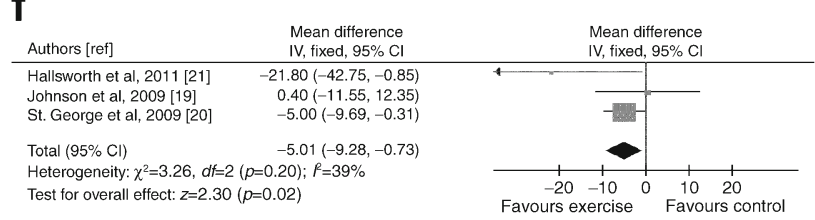

h

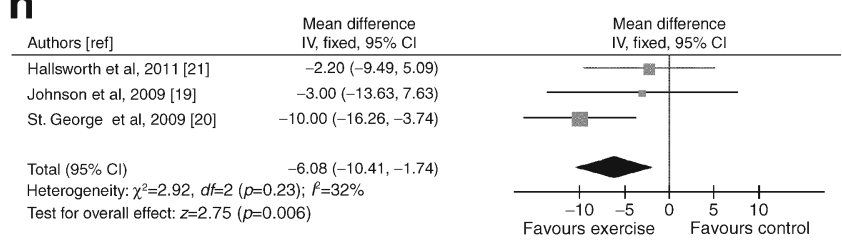

i

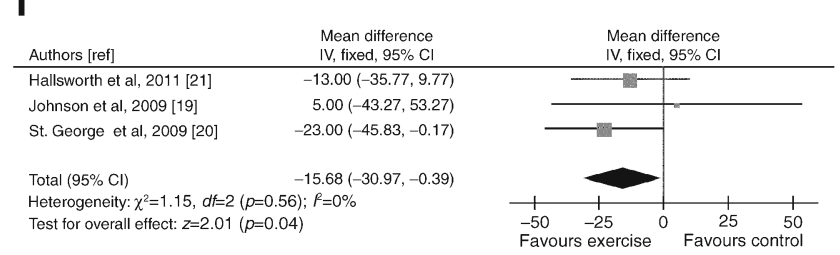

Fig. 3 Forest plots of RCTs comparing the effect of physical exercise alone on liver disease, glucose metabolism and cardiovascular risk. (a) NMR-assessed liver fat change (\%). (b) ALT change (IU/1). (c) Body weight change (\%). (d) Waist circumference change (\%).

turn improved LDL-C and HDL-C more consistently than the low-carbohydrate diet (Fig. 4).

These studies suggest that caloric restriction is the most important goal for improving hepatic steatosis, but a different nutrient composition may carry additional benefits according to individual patient features.

Insulin-sensitisers: thiazolidinediones

Thiazolidinediones (TZDs) were evaluated in 11 RCTs ( 862 participants, $38 \%$ diabetic; seven RCTs with low risk of bias) [26-37] (ESM Table 2).

Liver disease Pooled results of seven RCTs with posttreatment histology showed that TZDs improved steatosis, hepatocellular ballooning and inflammation but not fibrosis; (e) HOMA index change (\%). (f) FPG change (\%). (g) $\mathrm{HbA}_{\mathrm{lc}}$ change (\%). (h) Plasma LDL-cholesterol change (\%). (i) Plasma TG change (\%).To convert values for $\mathrm{HbA}_{1 \mathrm{c}}$ in $\%$ into $\mathrm{mmol} / \mathrm{mol}$, subtract 2.15 and multiply by 10.929 . IV, inverse variance

however, when considering patients with improved or stable fibrosis stage versus those with worsening fibrosis stage, TZDs significantly reduced the risk of fibrosis progression (Fig. 5). Heterogeneity was low for all assessed outcomes, suggesting a consistent drug effect size across studies. There was no significant publication bias (ESM Fig. 2)

Presence/absence of diabetes, the implementation of lifestyle intervention, different drug, dose or trial duration and risk of bias did not affect outcomes.

Glucose metabolism and cardiovascular risk TZDs improved HOMA, FPG, $\mathrm{HbA}_{1 \mathrm{c}}$, HDL-C, TG, CRP and adiponectin, but had no effect on LDL-C and BP (Fig. 5). TZDs improved also hepatic, muscle and adipose tissue insulin resistance $[26,34,37]$. There was no significant publication bias for assessed outcomes (not reported). 


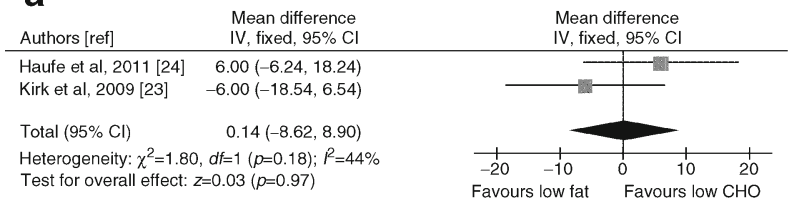

C

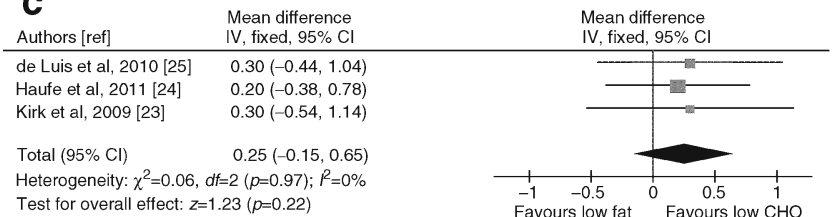

Test for overall effect: $z=1.23(p=0.22)$

\section{e}

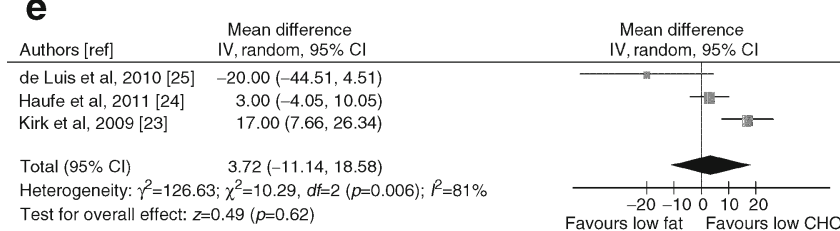

\section{g}

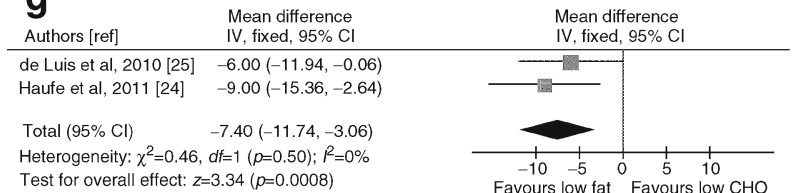

$$
\text { i }
$$

\begin{tabular}{|c|c|c|}
\hline Authors [ref] & $\begin{array}{l}\text { Mean difference } \\
\text { IV, random, } 95 \% \mathrm{Cl}\end{array}$ & $\begin{array}{l}\text { Mean difference } \\
\text { IV, random, } 95 \% \mathrm{Cl}\end{array}$ \\
\hline de Luis et al, 2010 [25] & $39.00(1.87,76.13)$ & $\longrightarrow$ \\
\hline Haufe et al, 2011 [24] & $3.00(-9.72,15.72)$ & - - \\
\hline Total $(95 \% \mathrm{Cl})$ & $16.60(-17.61,50.81)$ & \\
\hline $\begin{array}{l}\text { Heterogeneity: } \gamma^{2}=447.4 \\
\text { Test for overall effect: } z\end{array}$ & $\begin{array}{l}5 ; \chi^{2}=3.23, d f=1 \quad(p=0.07) ; P^{2}=69 \% \\
=0.95(p=0.34)\end{array}$ & $\begin{array}{ccccc}1 & 1 & 1 & 1 \\
-50 & -25 & 0 & 25 & 50 \\
\text { Favours low fat } & \text { Favours low } \mathrm{CHO}\end{array}$ \\
\hline
\end{tabular}

Fig. 4 Forest plots of RCTs comparing the effect of low fat versus low carbohydrate $(\mathrm{CHO})$ dietary caloric restriction on liver disease, glucose metabolism and cardiovascular risk. (a) NMR-assessed liver fat change (\%). (b) ALT change (IU/l). (c) Body weight change (\%). (d) Waist

For some outcomes heterogeneity was high: for LDL-C, heterogeneity was abated after excluding one RCT [30], showing unexpected LDL-C increase with rosiglitazone (weighed mean difference [WMD] 1.13, 95\% CI -2.40 , 4.66, $p=0.53, \mathrm{I}^{2}=34 \%, n$ comparisons $=5$ ). For HOMA, heterogeneity was abated after excluding one RCT [29], showing unexpected HOMA increase with pioglitazone (WMD $-33 \%, 95 \%$ CI $-44 \%,-22 \%, p=0.00001, \mathrm{I}^{2}=40 \%$, $n$ comparisons $=7$ ).

For BP, after excluding the only RCT using rosiglitazone [34], the remaining trials showed no change in systolic BP (WMD $-1.5 \%, 95 \%$ CI $-4.4 \%,-1.2 \%, p=0.27$, $\mathrm{I}^{2}=12 \%, n$ comparisons $=3$ ) or a reduction in diastolic BP (WMD $-3.3 \%, 95 \%$ CI $-5.5 \%,-1.0 \%, p=0.005, \mathrm{I}^{2}=0 \%$, $n$ comparisons $=3$ ) with pioglitazone.

For adiponectin, heterogeneity was abated after excluding two RCTs using a lower dose of pioglitazone [29] or did not b

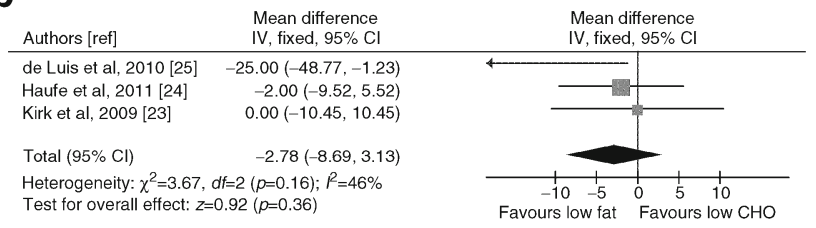

d

\begin{tabular}{|c|c|c|c|}
\hline \multirow{2}{*}{$\begin{array}{l}\text { Authors [ref] } \\
\text { de Luis et al 2010 [25] }\end{array}$} & \multirow{2}{*}{$\begin{array}{l}\text { Mean difference } \\
\text { IV, fixed, } 95 \% \mathrm{Cl}\end{array}$} & \multicolumn{2}{|c|}{$\begin{array}{l}\text { Mean difference } \\
\text { IV, fixed, } 95 \% \mathrm{Cl}\end{array}$} \\
\hline & & & \\
\hline Haufe et al, 2011 [24] & $0.50(-0.08,1.08)$ & & - \\
\hline Kirk et al, 2009 [23] & $1.00(-0.67,2.67)$ & & \\
\hline Total $(95 \% \mathrm{Cl})$ & $0.64(0.11,1.18)$ & & \\
\hline \multicolumn{2}{|c|}{$\begin{array}{l}\text { Heterogeneity: } \chi^{2}=2.91, d f=2(p=0.23) ; P^{2}=31 \% \\
\text { Test for overall effect: } z=2.36(p=0.02)\end{array}$} & $\begin{array}{lc}-2 & -1 \\
\text { Favours low fat }\end{array}$ & ${ }^{1}{ }^{1}{ }^{2}{ }^{2}$ \\
\hline
\end{tabular}

$\mathbf{f}$

\begin{tabular}{|c|c|c|c|}
\hline Authors [ref] & $\begin{array}{l}\text { Mean difference } \\
\text { IV, fixed, } 95 \% \mathrm{Cl}\end{array}$ & \multicolumn{2}{|c|}{$\begin{array}{l}\text { Mean difference } \\
\text { IV, fixed, } 95 \% \mathrm{Cl}\end{array}$} \\
\hline de Luis et al, 2010 [25] & $13.00(0,37,25.63)$ & & \\
\hline Haufe et al, 2011 [24] & $10.30(3.36,17.24)$ & & $\longrightarrow-$ \\
\hline Kirk et al, 2009 [23] & $2.70(-4.82,10.22)$ & & \\
\hline Total $(95 \% \mathrm{Cl})$ & $7.67(2.95,12.40)$ & & \\
\hline \multicolumn{2}{|c|}{$\begin{array}{l}\text { Heterogeneity: } \chi^{2}=2.91, d f=2(p=0.23) ; r^{2}=31 \% \\
\text { Test for overall effect: } z=3.18(p=0.001)\end{array}$} & $\begin{array}{cc}-20 & -10 \\
\text { Favours low fat }\end{array}$ & $\begin{array}{lcc} & 1 & 1 \\
0 & 10 & 20 \\
\text { Favours low } & \mathrm{CHO}\end{array}$ \\
\hline
\end{tabular}

h

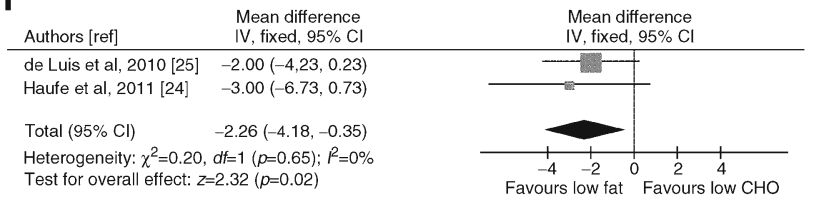

j

\begin{tabular}{lcc} 
Authors [ref] & $\begin{array}{c}\text { Mean difference } \\
\mathrm{IV}, \text { fixed, } 95 \% \mathrm{Cl}\end{array}$ & $\begin{array}{c}\text { Mean difference } \\
\text { IV, fixed, } 95 \% \mathrm{Cl}\end{array}$ \\
\hline $\begin{array}{l}\text { Haufe et al, } 2011[24] \\
\text { Kirk et al, } 2009[23]\end{array}$ & $-14.00(-32.84,4.84)$ & \\
& $0.00(-8.04,8.04)$ &
\end{tabular}

circumference change (\%). (e) HOMA index change (\%). (f) FPG change (\%). (g) Plasma LDL-cholesterol change (\%). (h) Plasma HDL-cholesterol change (\%) (i) Plasma TG change (\%). (j) Serum adiponectin change $(\%)$. IV, inverse variance

vigorously implement lifestyle intervention [30] (WMD 118\%, $95 \%$ CI 82, 155, $p=0.00001, I^{2}=0 \%, n$ comparisons $=3$ ).

Weight gain (mean $2 \%$, range $0-4.8 \%$ ) occurred in up to $75 \%$ of patients, accompanied by an increased in waist circumference, and was a common cause of dropout, together

Fig. 5 Forest plots of RCTs comparing the effect of thiazolidinedione on liver disease, glucose metabolism and cardiovascular risk. (a) Improvement in histological steatosis in NASH. (b) Improvement in lobular inflammation in NASH. (c) Improvement in hepatocellular ballooning in NASH. (d) Improvement in fibrosis in NASH. (e) Improvement or stability in fibrosis in NASH. (f) Body weight change (\%). (g) Waist circumference change (\%). (h) Systolic BP changes $(\mathrm{mmHg})$. (i) Diastolic BP changes (mmHg). (j) HOMA index change (\%). (k) FPG change (\%). (l) $\mathrm{HbA}_{\mathrm{lc}}$ change (\%). (m) Plasma LDL-cholesterol change (\%). (n) Plasma HDL-cholesterol change (\%) (o) Plasma TG change (\%). (p) Serum C-reactive protein change $(\mathrm{mg} / \mathrm{l})$. (q) Serum adiponectin change (\%). To convert values for $\mathrm{HbA}_{1 \mathrm{c}}$ in $\%$ into $\mathrm{mmol} / \mathrm{mol}$, subtract 2.15 and multiply by 10.929 . M-H, Mantel-Haenszel 


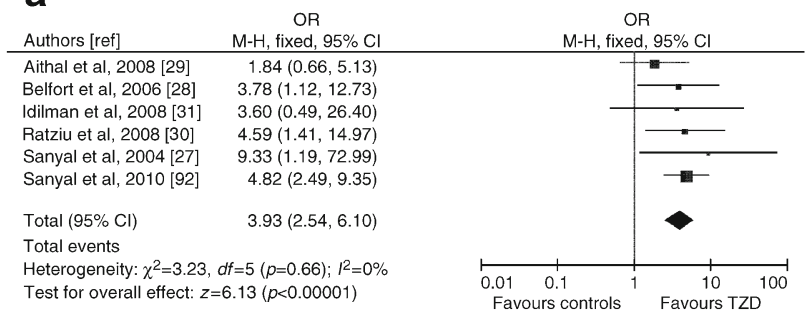

C

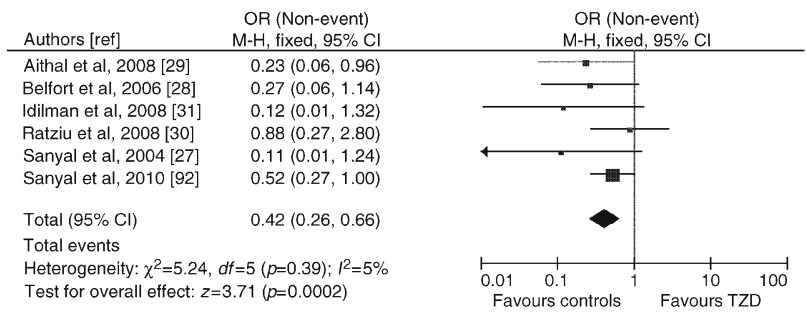

e

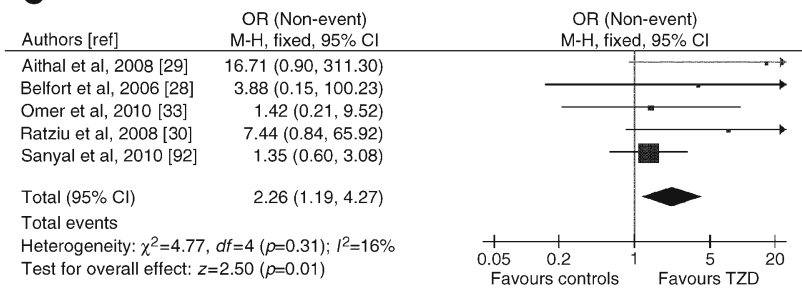

\section{g}

\begin{tabular}{|c|c|c|c|}
\hline Authors [ref] & $\begin{array}{l}\text { Mean difference } \\
\text { IV, fixed, } 95 \% \mathrm{CI}\end{array}$ & $\begin{array}{l}\text { Mean diff } \\
\text { IV, fixed, }\end{array}$ & $\begin{array}{l}\text { erence } \\
95 \% \mathrm{Cl}\end{array}$ \\
\hline Aithal et al, 2008 [29] & $1.03(-2.80,4.86)$ & & \\
\hline Gupta et al, 2010 [37] & $1.28(0.79,1.77)$ & & it \\
\hline Idilman et al, 2008 [31] & $4.70(1.97,7.43)$ & & \\
\hline Omer et al, 2010 [33] & $1.50(-0.92,3.92)$ & & \\
\hline Sanyal et al, 2004 [27] & $0.50(-1.73,2.73)$ & & \\
\hline $\begin{array}{l}\text { Sanyal et al, } 2010 \text { [92] } \\
\text { Tiikkainen et al, } 2004 \text { [34] }\end{array}$ & $\begin{array}{r}2.90(-0.17,5.97) \\
2.90(0.96,484)\end{array}$ & & \\
\hline Total $(95 \% \mathrm{Cl})$ & $1.46(1.02,1.91)$ & & 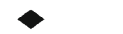 \\
\hline $\begin{array}{l}\text { Heterogeneity: } \chi^{2}=9.64, d f= \\
\text { Test for overall effect: } z=6 \text {. }\end{array}$ & $\begin{array}{l}=6(p=0.14) ; 1^{2}=38 \% \\
.45(p<0.00001)\end{array}$ & $\begin{array}{lcl}1 & 1 & 1 \\
-4 & -2 & 0 \\
\text { Favours control }\end{array}$ & $\begin{array}{cc}1 & 1 \\
2 & 4 \\
\text { Favours TZD }\end{array}$ \\
\hline
\end{tabular}

i

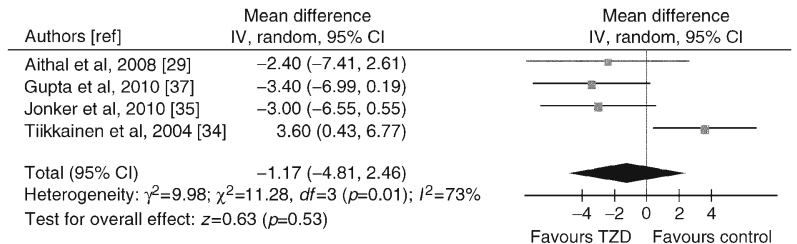

\section{k}

\begin{tabular}{|c|c|c|c|}
\hline Authors [ref] & $\begin{array}{l}\text { Mean difference } \\
\text { IV, fixed, } 95 \% \mathrm{Cl}\end{array}$ & $\begin{array}{l}\text { Mean } \\
\text { IV, fixe }\end{array}$ & $\begin{array}{l}\text { difference } \\
\text { d, } 95 \% \mathrm{Cl}\end{array}$ \\
\hline Aithal et al, 2008 [29] & $-14.00(-24.48,-3.52)$ & & \\
\hline Belfort et al, 2006 [28] & $-18.00(-32.95,-3.05)$ & & \\
\hline Gupta et al, 2010 [37] & $-5.90(-13.07,1.27)$ & & \\
\hline Idilman et al, 2008 [31] & $-14.80(-22.09,-7.51)$ & & \\
\hline Omer et al, 2010 [33] & $-13.10(-23.39,-2.81)$ & & \\
\hline Ratziu et al, 2008 [30] & $-16.00(-30.82,-1.18)$ & & \\
\hline Sanyal et al, 2010 [92] & $-5.30(-11.13,0.53)$ & & \\
\hline Total $(95 \% \mathrm{Cl})$ & $-10.02(-13.26,-6.77)$ & & \\
\hline \multicolumn{2}{|c|}{$\begin{array}{l}\text { Heterogeneity: } \chi^{2}=8.05, d f=6(p=0.23) ; l^{2}=25 \% \\
\text { Test for overall effect: } z=6.06(p<0.00001)\end{array}$} & $\begin{array}{ll}-20 & -10 \\
\text { Favours TZD }\end{array}$ & $\begin{array}{lll} & 1 & 1 \\
0 & 10 & 20 \\
\text { Favours control }\end{array}$ \\
\hline
\end{tabular}

b

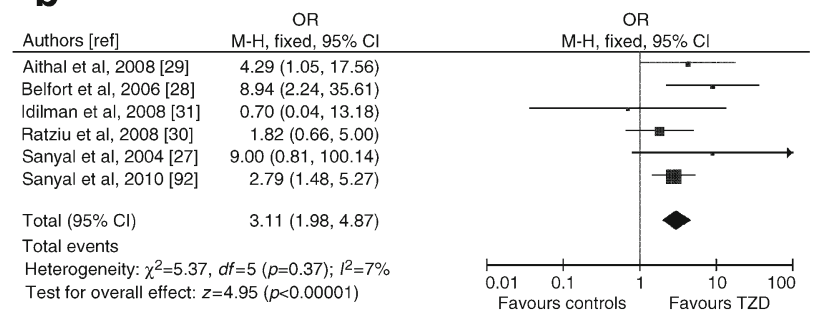

\section{d}

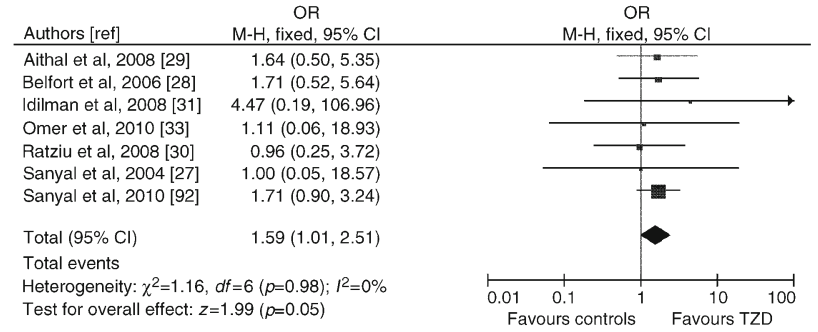

f

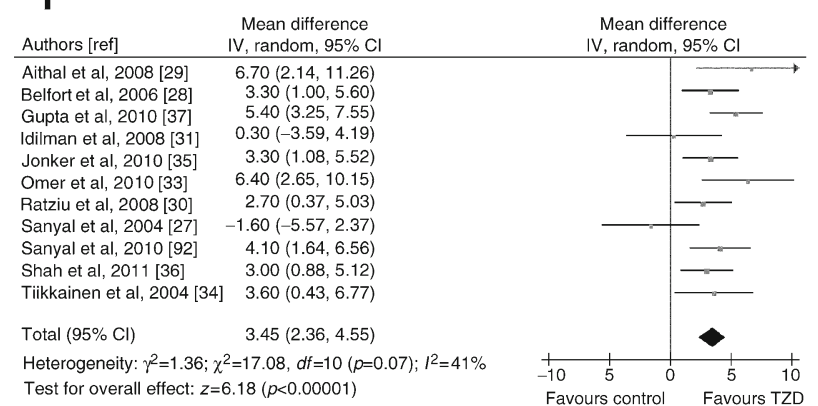

Test for overall effect: $z=6.18(p<0.00001)$

\section{h}

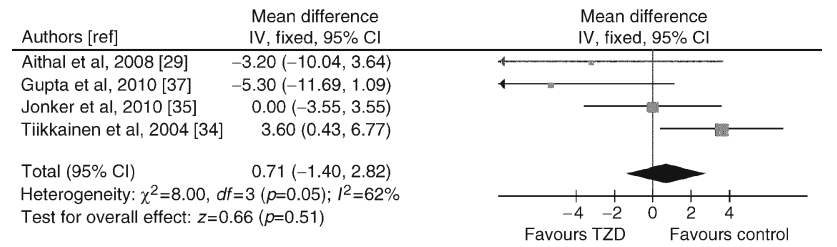

\section{j}

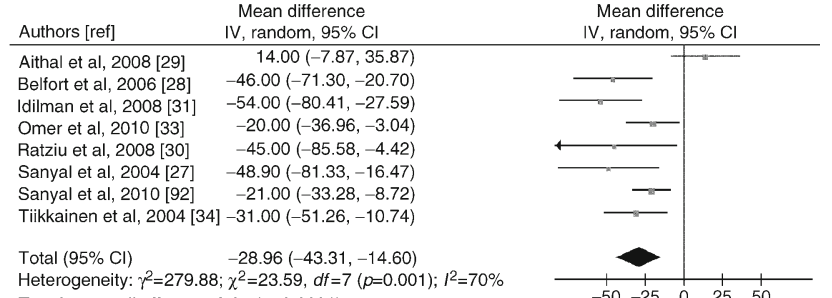
Test for overall effect: $z=3.95(p<0.0001)$

Favours TZD Favours control

I

\begin{tabular}{lr} 
Authors [ref] & $\begin{array}{c}\text { Mean difference } \\
\text { IV, fixed, 95\% Cl }\end{array}$ \\
\hline Aithal et al, 2008 [29] & $-0.30(-0.50,-0.10)$ \\
Belfort et al, 2006 [28] & $-0.60(-1.00,-0.20)$ \\
Omer et al, 2010 [33] & $-0.20(-0.44,0.04)$ \\
Ratziu et al, 2008 [30] & $-0.35(-0.74,0.04)$ \\
Shah et al, 2011 [36] & $0.10(-0.68,0.88)$ \\
& $-0.38(-1.43,-0.16)$
\end{tabular}

Total $(95 \% \mathrm{Cl}) \quad-0.38(-1.43,-0.16)$ Heterogeneity: $\chi^{2}=3.85, d f=4(p=0.43) ; 1^{2}=0 \%$ Test for overall effect: $z=4.37(p<0.0001)$

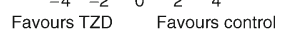


m

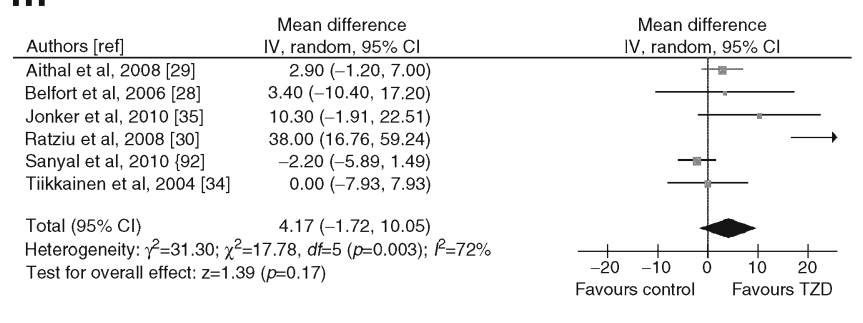

$\mathbf{0}$

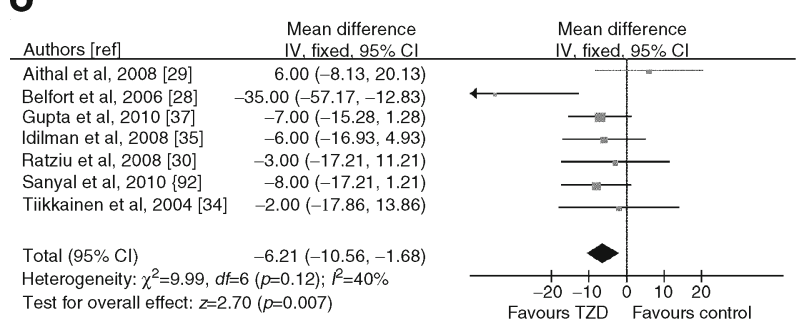

$\mathbf{q}$

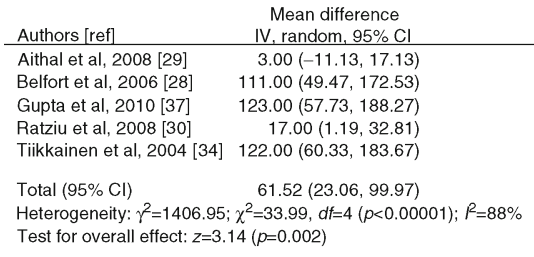

Fig. 5 (continued)

with ankle oedema (4-25\%). Weight gain did not reverse with treatment discontinuation and was not prevented by lifestyle intervention, but was reduced by metformin coadministration [33, 38]. Besides limiting weight gain, the combination of rosiglitazone + metformin offered no significant histological or cardio-metabolic benefit over rosiglitazone alone [33, 38].

NASH and associated cardio-metabolic abnormalities relapsed 1 year after discontinuing TZDs [38], posing the issue of the required treatment duration and of the benefit/safety of sustained thiazolidinedione treatment. In the Pioglitazone Versus Vitamin E versus Placebo for the Treatment of Nondiabetic Patients with NASH (PIVENS) and the Fatty Liver Improvement with Rosiglitazone Therapy (FLIRT)-2 trial, liver histology did not improve further despite continued HOMA and transaminase improvement over 2 and 3 years, respectively $[32,39]$. These two trials suggest that prolonged treatment with TZDs may offer no additional histological benefit and that metabolic improvement does not necessarily parallel histological improvement.

Due to the short trial duration, no cases of congestive heart failure, bone fractures or CVD events were reported. Concern about cardiovascular safety led the European Medicines Agency to recommend withdrawal of rosiglitazone from clinical use.
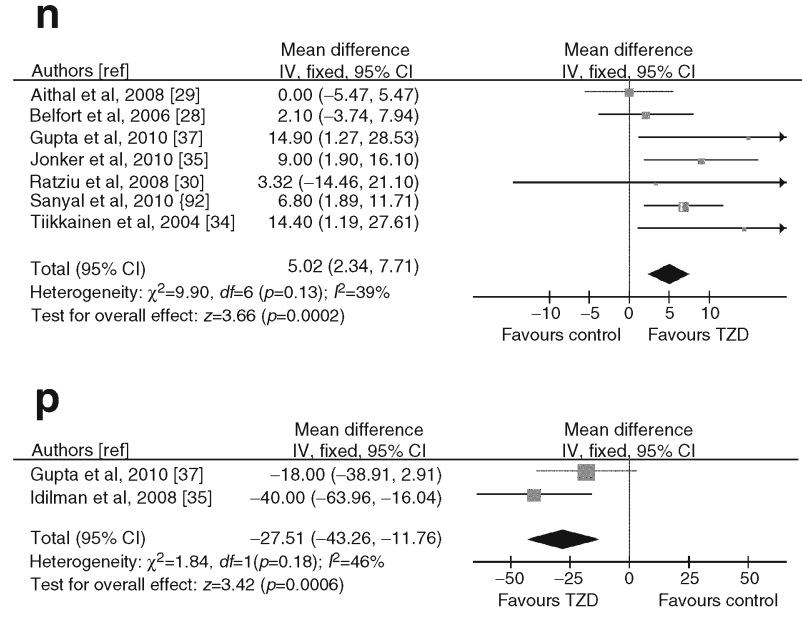

Insulin-sensitisers: metformin

Metformin has anorexigenic and weight-loss properties, decreases gastrointestinal glucose absorption and increases insulin sensitivity by increasing glucose uptake and AMPkinase-mediated oxidative glucose and lipid metabolism [40].

Eleven RCTs (671 participants, 27\% diabetic; six RCTs in NASH with post-treatment histology, three with a low bias risk) evaluated metformin [33, 34, 41-49] (ESM Table 2).

Liver disease Metformin did not improve liver histology compared with placebo (Fig. 6). Dose, treatment duration (ranging from 6 to 24 months) or diabetic state had no effect on post-treatment histology. There was no significant publication bias (ESM Fig. 2)

Fig. 6 Forest plots of RCTs comparing the effect of metformin on liver disease, glucose metabolism and cardiovascular risk. (a) Improvement in histological steatosis in NASH. (b) Improvement in lobular inflammation in NASH. (c) Improvement in hepatocellular ballooning in NASH. (d) Improvement in fibrosis in NASH. (e) Body weight change (\%). (f) Waist circumference change (\%). (g) HOMA index change (\%). (h) FPG change (\%). (i) $\mathrm{HbA}_{\mathrm{lc}}$ change (\%). (j) Plasma LDL-cholesterol change (\%). (k) Plasma HDL-cholesterol change (\%) (l) Plasma TG change (\%). (m) Serum C-reactive protein change (mg/l). (n) Serum adiponectin change $(\%)$. To convert values for $\mathrm{HbA}_{1 \mathrm{c}}$ in $\%$ into $\mathrm{mmol} / \mathrm{mol}$, subtract 2.15 and multiply by 10.929 . M-H, Mantel-Haenszel 
a

\begin{tabular}{|c|c|}
\hline Authors [ref] & M-H, fixed, $95 \% \mathrm{Cl}$ \\
\hline Haukeland et al, 2009 [43] & $0.56(0.15,2.05)$ \\
\hline 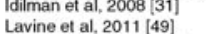 & $\begin{array}{l}2.00(0.26,15.38) \\
1.60(0.71,3.57)\end{array}$ \\
\hline Shields et al, 2009 [44] & $0.54(0.08,3.53)$ \\
\hline Uygun et al, $2004[42]$ & $5.25(1.09,25.21)$ \\
\hline Total $(95 \% \mathrm{Cl})$ & $1.42(0.82,2.46)$ \\
\hline
\end{tabular}

C

Authors [ref]

$O R$

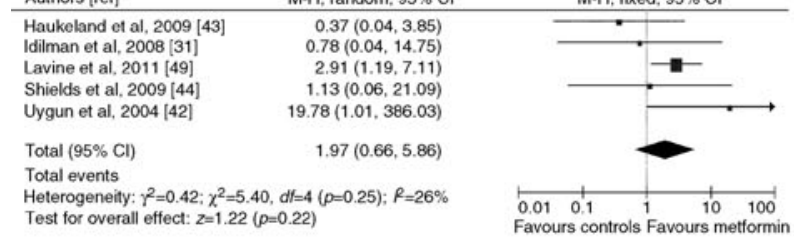

\begin{tabular}{l} 
e \\
Authors [ref] \\
\hline Bugianesi et al
\end{tabular}

Bugianesi et al, 2005 [45]

Garinis et al, $2010[47]$

Haukeland et al, 2009 (dilman et al, 2008 [31]
(a)

Lavine et al, 2011 [49]

Nar et al, $2009[46]$

Omer et al, 2010 [33]

Shields et al, 2009 [44]

Tock et al, $2010[48]$

Uygun et al, 2004 [42]

Total $(95 \% \mathrm{Cl})$

Test for overall effect: $z=5.60$ ( $p<0.00001)$

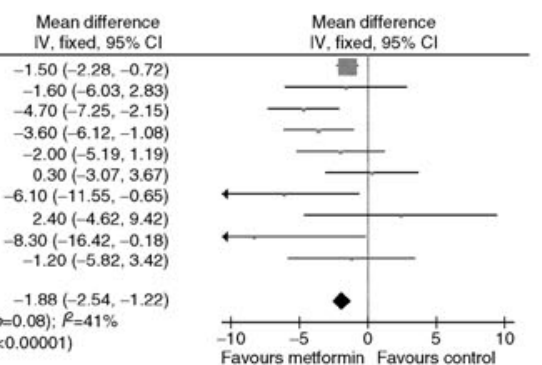

\section{g}

\section{Authors [ref]} Mean difference

Bugianesi et al, 2005 [45] Garinis et al, 2010 [47] Haukeland et al, $2009[43]$ Idilman et al, 2008 [31] Lavine et al. 2011 [49] Nar et al, 2009 [46] Shields et al, 2009 [44] Sofer et al, $2011(50]$ Tock et al, 2010 [48] Uygun et al, 2004 [42] IV, random, $95 \% \mathrm{Cl}$ $-19.00(-30.51,-7.49)$ $-14.00(-22.32,-5.68)$ $-1.00(-4.68,2.68)$ $45.00(-71.89,-18.11)$ $13.00(5.74,20.26)$ $10.00(0.31,19.69)$ $2.00(-25.02 .29 .02)$ $-14.00(-34.25,6.25)$ $34.00(-65.11,-2.89)$ $-44.00(-71.56,-16.44)$ Total $(95 \% \mathrm{Cl}) \quad-7.02(-13.82,-0.22)$ Heterogeneity: $\gamma^{2}=79.45 ; \chi^{2}=64.13, d t=10(p<0.00001) ; \quad R=84 \%$ Test for overall effect: $z=2.02(p=0.04)$

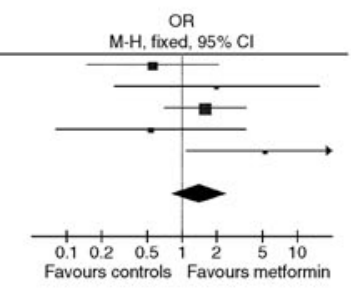

b \begin{tabular}{lrr} 
& Weight & M-H, fixed, 95\% C \\
\hline Haukeland et al, 2009 [43] & $29.8 \%$ & $0.35(0.08,1.57)$
\end{tabular} Idilman et al, $2008[31] \quad 4.8 \% \quad 0.78(0.04,14.75)$ Lavine et al, 2011 [49] $\quad 53.7 \% \quad 1.15(0.52 .2 .57)$ $\begin{array}{lrr}\text { Shields et al, } 2009[44] & 10.2 \% & 1.20(0.19,7.44) \\ \text { Uygun et al, 2004 [42] } & 1.4 \% & 18.20(0.88,374.89)\end{array}$ Total $(95 \% \mathrm{Cl}) \quad 100.0 \% \quad 1.15(0.64,2.06)$

Total events

Heterogeneity: $\chi^{2}=5.67, d t=4(p=0.23) ; \quad R=29 \%$

Test for overall effect: $z=0.45(p=0.65)$

d

f

\begin{tabular}{lr} 
Authors [ref] & $\begin{array}{c}\text { Mean difference } \\
\text { IV, fixed, 95\% Cl }\end{array}$ \\
\hline Garinis et al, 2010 [47] & $0.50(-5.04,6.04)$ \\
Idilman et al, 2008 [31] & $-4.20(-7.56,-0.84)$ \\
Lavine et al, 2011 [49] & $-1.50(-5.12,2.12)$ \\
Nar et al, 2009 [46] & $3.40(-1.34,8.14)$ \\
Omer et al, 2010 [33] & $-1.50(-3.32,0.32)$ \\
Tock et al, 2010 [48] & $-3.00(-13.14,7.14)$ \\
Total $(95 \% \mathrm{Cl})$ & $-1.45(-2.79,-0.10)$ \\
Heterogeneity: $\chi^{2}=7.16, d t=5(p=0.21) ; R=30 \%$ \\
Test for overall effect: $z=2.11(p=0.03)$
\end{tabular}

Heterogeneity: $\chi^{2}=7.16, d t=5(p=0.21) ;$
Test for overall effect: $z=2.11 \quad(p=0.03)$

h

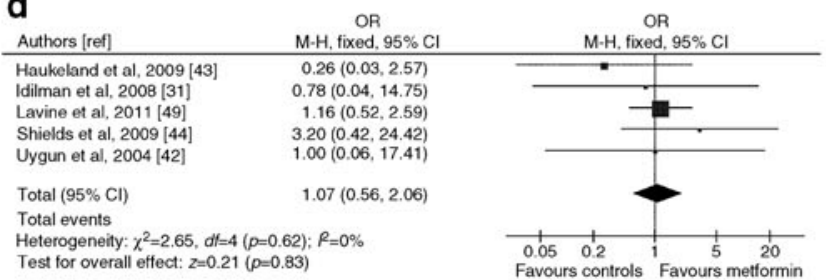
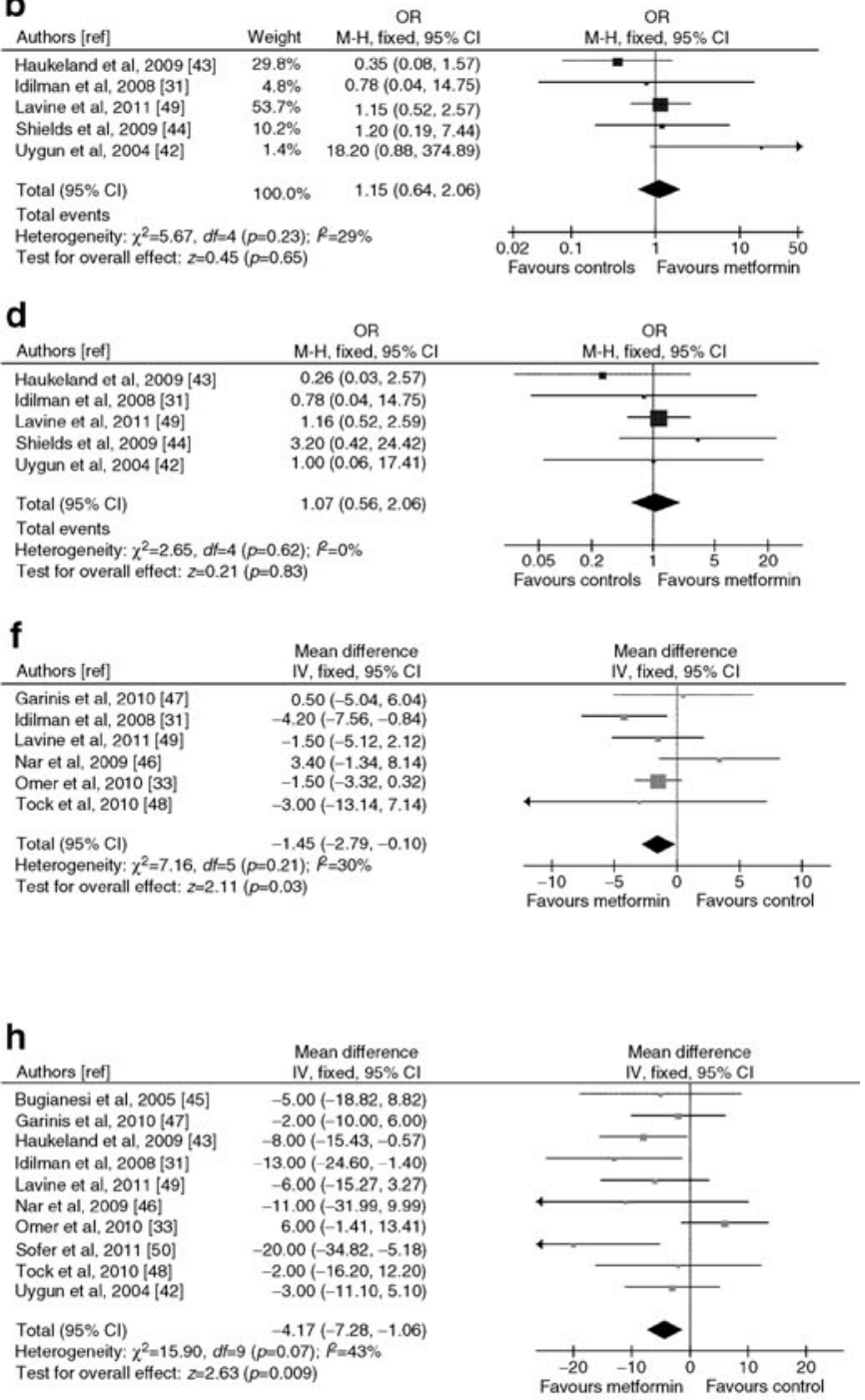

\section{j Authors [ref] Garinis et al, 2010 [47] Haukeland et al, 2009 [43] Lavine et al, 2011 [49] Nar et al, $2009[46]$
Sofer et al, 2011 [50] Total $(95 \% \mathrm{Cl})$
Heterogeneity: $\chi^{2}=6.60, d t=4(p=0.16) ; \quad(-8.95,0.76)$ Test for overall effect: $z=1.65(p=0.10)$

Mean difference
IV. fixed, $95 \% \mathrm{Cl}$
$4.00(-14.29,22.29)$
$-15.00(-31.62,1.62)$
$-3.00(-11.60,5.60)$
$-12.00(-22.15,-1.85)$
$2.00(-6.89,10.89)$
$-4.10(-8.95,0.76)$
$-0.16) ; R=39 \%$
$=0.10)$

\section{I}

Authors [ref] Garinis et al, 2010 [47] Haukeland et al, 2009 [43] Idilman et al, 2008 [31] Lavine et al, 2011 [ 46$]$ Nar et al, 2009 [50] Sofer et al, 2011 [42]
Uygun et al, 2004 Total $(95 \% \mathrm{Cl})$
Heterogeneity: $x^{2}=10.09, d f=6(p=0.12): R=41 \%$ Test for overall effect: $z=1.09(p=0.27)$

\section{Mean difference
IV. fixed. $95 \% \mathrm{Cl}$ $7.00(-9.63,23.63)$ $2.00(-8.68,12.68)$ $-8.00(-17.52,1.52)$ $-10.60(-29.78,8.58)$ $12.00(-8.31,32.31)$ $5.00(-28.34,-1.66)$ $5.00(-10.69,20.69)$}

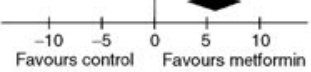

\section{m}

\begin{tabular}{lr} 
Muthors [ref] & $\begin{array}{c}\text { Mean difference } \\
\text { IV, fixed, } 95 \% \mathrm{Cl}\end{array}$ \\
\hline Garinis et al, 2010 [47] & $-1.20(-2.73,0.33)$ \\
Haukeland et al, 2009 [43] & $0.30(-1.48,2.08)$ \\
Idilman et al, 2008 [31] & $-3.10(-5.88,-0.32)$ \\
Sofer et al, $2011[50]$ & $0.00(-1.98,1.98)$ \\
Total $(95 \% \mathrm{Cl})$ & $-0.73(-1.67,0.22)$ \\
Heterogeneity: $\chi^{2}=4.97, d f=3(p=0.17) ; R=40 \%$ \\
Test for overall effect: $z=1.51(\rho=0.13)$
\end{tabular}

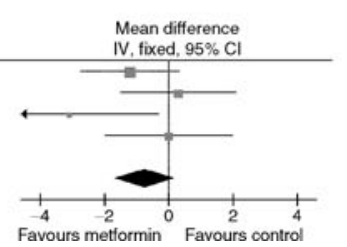

n

\begin{tabular}{|c|c|c|c|}
\hline $\begin{array}{l}\mathbf{n} \\
\text { Authors [ref] }\end{array}$ & $\begin{array}{l}\text { Mean difference } \\
\text { IV. fixed. } 95 \% \mathrm{Cl}\end{array}$ & $\begin{array}{l}\text { Mean } \\
\text { IV, fixe }\end{array}$ & $\begin{array}{l}\text { difference } \\
\text { ed, } 95 \% \mathrm{Cl}\end{array}$ \\
\hline Garinis et al, 2010 [47] & $13.10(3.68,22.52)$ & & $\longrightarrow$ \\
\hline Haukeland et al, 2009 [43] & $-0.10(-11.03,10.83)$ & & \\
\hline Sofer et al, 2011 [50] & $11.20(0.33,22.07)$ & & \\
\hline Total $(95 \% \mathrm{Cl})$ & $8.60(2.63,14.56)$ & & \\
\hline $\begin{array}{l}\text { Heterogeneity: } \chi^{2}=3.53, d f= \\
\text { Test for overall effect: } z=2.8\end{array}$ & $\begin{array}{l}p=0.17) ; R=43 \% \\
p=0.005)\end{array}$ & $\begin{array}{ll}-20 & -10 \\
\text { Favours control }\end{array}$ & $\begin{array}{l}0 \\
0 \\
\text { Favours metformin }\end{array}$ \\
\hline
\end{tabular}


Glucose metabolism and cardiovascular risk Metformin significantly reduced body weight, waist circumference, HOMA, FPG, and HbAlc, and increased HDL-C and adiponectin, but had no effect on LDL-C, TG, blood pressure [50] and CRP (Fig. 2). There was no significant publication bias for assessed outcomes (not reported).

Heterogeneity of results for HOMA was abated after excluding trials not effectively implementing lifestyle intervention (as suggested by absence of weight loss in the controls) $[43,46,49]$ (WMD $-21 \%, 95 \%$ CI $-31,-11$, $p=0.0001, I^{2}=40 \%, n$ comparisons $\left.=7\right)$, suggesting that the insulin-sensitising effects of metformin are potentiated when lifestyle intervention is effectively implemented.

Metformin was safe and well-tolerated: gastrointestinal intolerance was the most common adverse effect, not requiring treatment discontinuation.

Lipid-lowering drugs

Statins The hepatological safety of statins in NAFLD has been recently recognised and their feared potential for worsening glucose tolerance seems largely outweighed by their cardiovascular benefit $[50,51]$.

Four RCTs (719 participants, three with a low bias risk) assessed statins in NAFLD [52-55] (ESM Table 3).

Liver disease Statins improved ALT (ESM Fig. 3) and radiological steatosis $[53,54]$ in hyperlipidaemic NAFLD patients; in the only RCT with post-treatment histology, simvastatin had no effect on liver histology [56]. There was no significant publication bias (ESM Fig. 2)

Glucose metabolism and cardiovascular risk Statins improve LDL-C, HDL-C and TG without affecting body weight (ESM Fig. 3). One RCT found no effect of statins on waist circumference, BP, FPG and CRP [53]. There was no significant publication bias for assessed outcomes (not reported).

In a post hoc analysis of the Greek Atorvastatin and Coronary Heart Disease Evaluation (GREACE) RCT, stain-treated hyperlipidaemic NAFLD patients experienced a significant $(-68 \%)$ risk reduction of CVD events compared with both presumed NAFLD patients not on statin and with statin-treated patients with normal transaminases, without significant adverse events, including new-onset diabetes [55]. Importantly, this study demonstrates that statins are safe in NAFLD and that statin-related cardiovascular benefit is greater in patients with NAFLD than in those with normal liver tests.

Ezetimibe Growing evidence connects non-esterified cholesterol accumulation in mitochondria to hepatocyte apoptosis, liver injury and NASH development [56-61]. On this basis, ezetimibe, a Niemann-Pick C1 like 1 protein inhibitor, was evaluated in two RCTs in NAFLD.

Liver disease Ezetimibe reduced histological ballooning and fibrosis in one RCT, and MR-assessed liver fat in the other [62, 63] (ESM Table 3).

Glucose metabolism and cardiovascular risk Ezetimibe improved LDL-C and CRP, without affecting weight, waist and HOMA. In one RCT, ezetimibe treatment was associated with significant $\mathrm{HbA}_{1 \mathrm{c}}$ increase compared with placebo [64].

n-3 polyunsaturated fatty acids Five RCTs (303 participants, two RCTs with low risk of bias) evaluated polyunsaturated fatty acids (PUFA) [64-68] (ESM Table 3).

Liver disease PUFA improved ALT (ESM Fig. 4) and steatosis by NMR or ultrasound [65-68]. In the only RCT with post-treatment histology, PUFA ameliorated steatosis without affecting other histological features [68]. There was no significant publication bias (ESM Fig. 2).

Glucose metabolism and cardiovascular risk PUFA ameliorated HOMA, HDL-C and TG, but had no effect on body weight, BP and LDL-C (ESM Fig. 4). One RCT found no effect of PUFA on waist circumference and CRP [68]. There was no significant publication bias for assessed outcomes (not reported).

Overall, PUFA were well-tolerated, with minor gastrointestinal symptoms.

Probucol Probucol, a lipophilic lipid-lowering agent with strong antioxidant activity, was evaluated in NASH in one RCT: ALT improved, but post-treatment histology was unavailable [69] (ESM Table 3). Although generally well-tolerated, probucol was associated with a significant fall in HDL-C.

Fibrates Following consistent anti-steatogenic activity in animal models of NAFLD [70], fibrates were evaluated in five RCTs (315 participants, four RCTs with a low risk of bias) $[53,71-74]$ (ESM Table 3$)$.

Liver disease Fibrates had no effect on ALT (ESM Fig. 5), radiological steatosis [75] or liver histology [73]. There was no significant publication bias (ESM Fig. 2).

Glucose metabolism and cardiovascular risk Fibrates improved HDL-C and TG, had no effect on body weight, waist, HOMA, FPG and LDL-C (ESM Fig. 5). One RCT showed no effect of fibrates on BP and adiponectin [53]. There was no significant publication bias for assessed outcomes (not reported). 
Angiotensin receptor blockers

The modulation of insulin sensitivity, systemic inflammation, hepatic lipogenesis and fibrogenesis by the renin-angiotensin system and the frequent coexistence of hypertension prompted evaluation of angiotensin receptor blockers in NAFLD. In a well-designed RCT on hypertensive NASH, telmisartan (an angiotensin receptor blocker with peroxisome proliferator activated receptor [PPAR]- $\gamma$-regulating activity) improved steatosis, necroinflammation, fibrosis, HOMA, TG and total cholesterol more consistently than valsartan, despite similar BP reduction, suggesting that the peculiar PPAR- $\gamma$-agonist activity may mediate the more consistent metabolic and histological benefits of telmisartan [75] (ESM Table 4).

In another RCT on hypertensive NAFLD patients, losartan plus simvastatin significantly improved ultrasonographic steatosis, visceral adiposity, HOMA and CRP compared with amlodipine plus simvastatin, despite similar BP reduction [76] (ESM Table 4).

\section{Endocannabinoid receptor antagonists}

The cannabinoid type 1 receptor (CB1) receptor antagonist rimonabant experimentally antagonised appetite, caloric intake, hepatic lipogenesis and fibrogenesis and was evaluated in abdominally obese, dyslipidaemic NAFLD patients from the ADAGIO-Lipids trial [77]: rimonabant reversed CT-assessed steatosis in $48 \%$ of patients versus $19 \%$ on placebo $(p=0.03)$ and extensively improved all cardio-metabolic variables (ESM Table 4). Depressive and anxiety disorders were more common with rimonabant

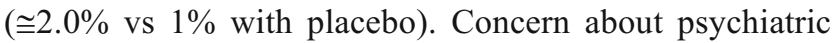
adverse effects led to withdrawal of rimonabant, but the development of peripherally acting CB1 antagonists is an area of intense research.

\section{Anti-TNF- $\alpha$ agents (pentoxifylline)}

The anti-TNF- $\alpha$ agent pentoxifylline has been evaluated in four RCTs in NASH [78-81] (three with low risk of bias, two assessing post-treatment histology) (ESM Table 4).

Liver disease Pooled data analysis showed that pentoxifylline improved histological steatosis and lobular inflammation (ESM Fig. 6). There was no significant publication bias (ESM Fig. 2).

Glucose metabolism and cardiovascular risk Pentoxifylline had no effect on body weight and HOMA (ESM Fig. 6). One RCT found no impact on plasma LDL-C, HDL-C and TG [80]. There was no significant publication bias for assessed outcomes (not reported).
Overall, pentoxifylline was safe and well-tolerated with minor gastrointestinal symptoms.

Ursodeoxycholic acid (UDCA)

Seven RCTs (639 participants, 21\% diabetic; three RCTs with post-treatment histology, five RCTs with a low risk of bias) evaluated UDCA in NAFLD (ESM Table 5) [82-88].

Liver disease Overall, UDCA improved ALT but not liver histology (Fig. 7). For ALT and for lobular inflammation, heterogeneity was high and was abated when considering RCT evaluating high-dose (twofold higher than the conventional dose) UDCA or UDCA + vitamin E, showing a modest benefit: for ALT WMD $-20 \mathrm{IU} / 1,95 \% \mathrm{CI}-37,-3, p=0.02$, $\mathrm{I}^{2}=40 \%, n$ comparisons $=3$; for lobular inflammation $\mathrm{OR} 2.3$; $95 \%$ CI 1.1, 5.0; $p=0.03, I^{2}=0 \%, n$ comparisons $\left.=2\right)$. There was no significant publication bias (ESM Fig. 2).

Glucose metabolism and cardiovascular risk UDCA improved adiponectin (Fig. 7). For HOMA and FPG heterogeneity was abated after excluding one RCT evaluating the combination of UDCA+vitamin E, the latter potentially worsening HOMA (see below), showing a consistent benefit with UDCA on both HOMA and FPG (for FPG: WMD $-6 \%$, $95 \%$ CI $-9,-2, p=0.0005, I^{2}=40 \%, n$ comparisons $=3$ ).

One RCT reported significant improvement in $\mathrm{HbA}_{1 \mathrm{c}}$ and HDL-C with high-dose UDCA [85]. There was no significant publication bias for assessed outcomes (not reported).

Minor gastrointestinal effects occurred in $>40 \%$ of patients on high-dose UDCA.

Semi-synthetic bile acids

Besides their role in nutrient absorption, bile acids are signalling molecules that exert genomic and non-genomic effects by activating TGR5 and farnesoid $\mathrm{X}$ receptor (FXR).

TGR5 is a G-protein-coupled receptor (expressed in brown adipose tissue muscle and gut), activation of which by bile acids increases energy expenditure and glucagonlike peptide-1 (GLP-1) secretion and attenuates diet-induced obesity [89, 90].

FXR is a nuclear hormone receptor expressed in the liver, intestine and kidney. In the liver, FXR controls lipogenesis, very-LDL metabolism, gluconeogenesis, glycogen synthesis and insulin sensitivity, and also has also anti-inflammatory and anti-fibrotic properties [90].

On this basis, a novel class of semi-synthetic bile acid agonists of TGR5/FXR is being evaluated for the treatment of obesity-related disorders, including NAFLD.

In the first RCT, Int-747, a semi-synthetic derivative of the human bile acid CDCA, administered for 6 weeks to 


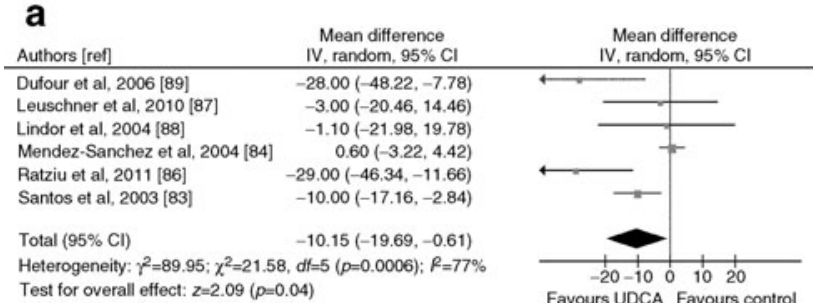

C

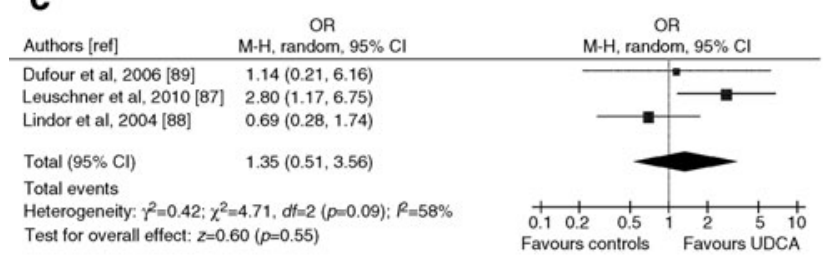

\section{e}

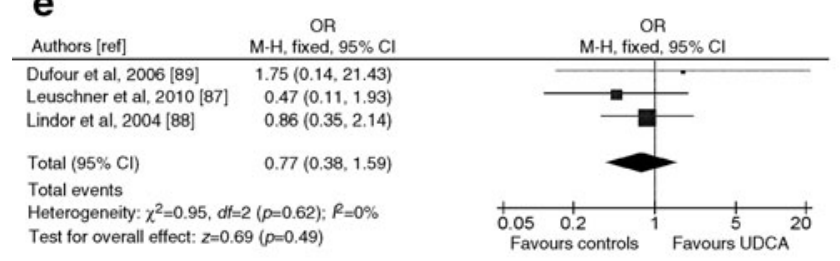

g

\begin{tabular}{|c|c|c|c|}
\hline Authors [ref] & $\begin{array}{l}\text { Mean difference } \\
\text { IV, random, } 95 \% \mathrm{CI}\end{array}$ & \multicolumn{2}{|c|}{$\begin{array}{l}\text { Mean difference } \\
\text { IV, random, } 95 \% \mathrm{Cl}\end{array}$} \\
\hline Dufour et al, 2006 [89] & $192.00(116.22,267.78)$ & & 二- \\
\hline Ratziu et al, 2011 [86] & $-26.00(-34.38,-17.62)$ & : & \\
\hline Total $(95 \% \mathrm{Cl})$ & $79.61(-133.92,293.15)$ & & \\
\hline $\begin{array}{l}\text { Heterogeneity: } x^{2}=2300 \\
\text { Test for overall effect: } z\end{array}$ & $\begin{array}{l}\chi^{2}=31.41, d f=1 \quad(p<0.00001) ; R=97 \% \\
(p=0.46)\end{array}$ & $\begin{array}{c}-200-100 \\
\text { Favours UDCA }\end{array}$ & $\begin{array}{lll}0 & 100 & 200 \\
0 & \text { Favours control }\end{array}$ \\
\hline
\end{tabular}

b

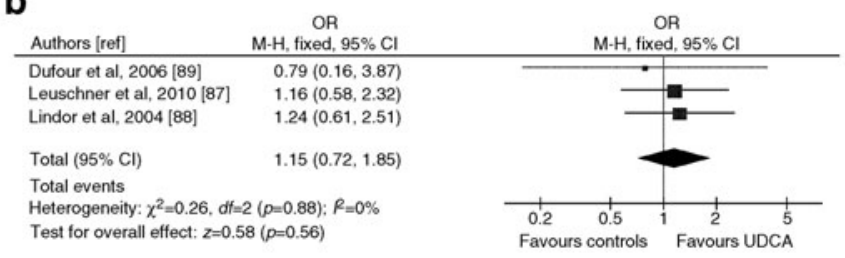

d

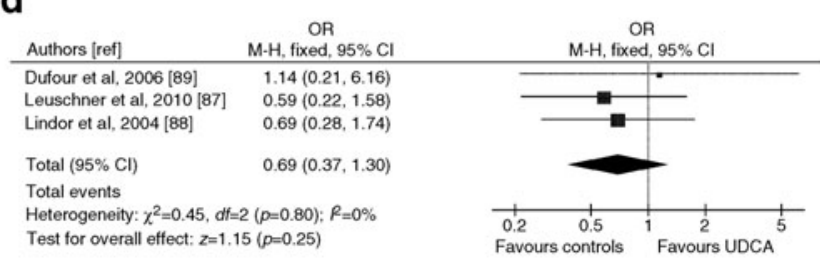

f

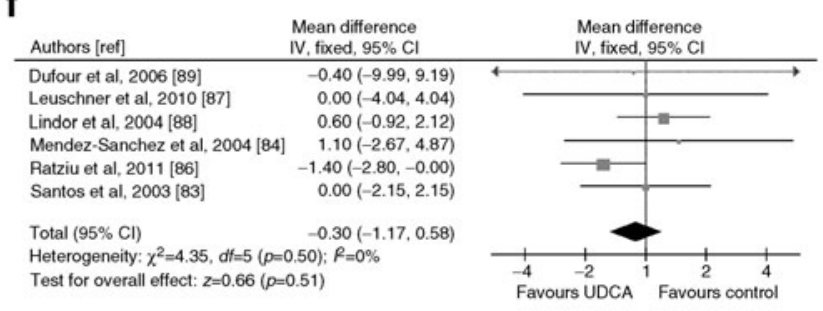

h

\begin{tabular}{|c|c|c|c|}
\hline \multirow{3}{*}{$\begin{array}{l}\text { Authors [ref] } \\
\text { Dufour et al, } 2006 \text { [89] }\end{array}$} & \multirow{2}{*}{$\begin{array}{c}\begin{array}{c}\text { Mean difference } \\
\text { IV, random, } 95 \% \mathrm{Cl}\end{array} \\
15.00(2.71,27.29)\end{array}$} & \multicolumn{2}{|c|}{$\begin{array}{c}\text { Mean difference } \\
\text { IV, random, } 95 \% \mathrm{CI}\end{array}$} \\
\hline & & & $\longrightarrow$ \\
\hline & $-2.00(-4.88,0.88)$ & & \\
\hline Mendez-Sanchez et al, 2004 [84] & $-9.00(-15.04,-2.96)$ & - & \\
\hline Ratziu et al, 2011 [86] & $-6.10(-7.85,-4.35)$ & $=$ & \\
\hline Total $(95 \% \mathrm{Cl})$ & $-3.20(-8.00,1.60)$ & & \\
\hline $\begin{array}{l}\text { Heterogeneity: } \gamma^{2}=16.62 ; \chi^{2}=17.54 \\
\text { Test for overall effect: } z=1.31 \quad(p=0\end{array}$ & $\begin{array}{l}\text { 4. } d f=3(p=0.0005) ; \quad R=83 \% \\
.19)\end{array}$ & $\begin{array}{lll}-20 & -10 & 0 \\
\text { Favours UDCA }\end{array}$ & $\begin{array}{cc}10 & 20 \\
\text { Favours control }\end{array}$ \\
\hline
\end{tabular}

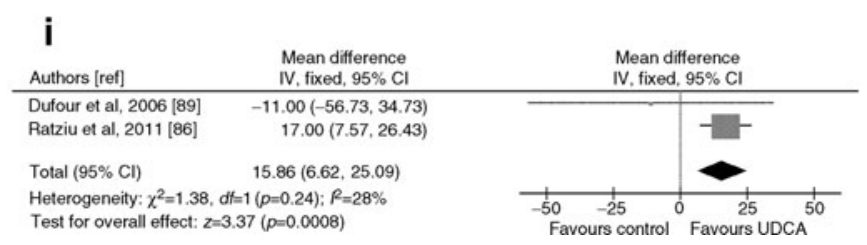

Fig. 7 Forest plots of RCTs comparing the effect of UDCA on liver disease, glucose metabolism and cardiovascular risk. (a) Improvement in serum ALT (IU/1). (b) Improvement in histological steatosis in NASH. (c) Improvement in lobular inflammation in NASH.

diabetic NAFLD patients, was well-tolerated, ameliorated markers of liver fibrosis, insulin resistance and induced weight loss compared with placebo (ESM Table 4) [91]. The ongoing FXR Ligand NASH Treatment (FLINT) double blind, placebo controlled, multicentre trial is evaluating the effects of obeticholic acid in NASH.

\section{Antioxidants}

Fifteen RCTs (1,141 participants, 9\% diabetic, seven RCTs with low risk of bias) evaluated antioxidants in NAFLD: overall, heterogeneity in study population, duration, type and (d) Improvement in hepatocellular ballooning in NASH. (e) Improvement in fibrosis in NASH. (f) Body weight change (\%). (g) HOMA index change (\%). (h) FPG change (\%). (i) Serum adiponectin change (\%). M-H, Mantel-Haenszel

dose of drug, lifestyle intervention implementation, was considerable [6, 32, 49, 92-99] (ESM Table 5).

Liver disease Pooled results of the seven RCTs (685 patients, 4\% diabetic) with post-treatment histology showed no histological improvement and high heterogeneity (Fig. 8). Heterogeneity was reduced when considering only the five RCTs with vitamin $\mathrm{E}$, showing modest improvement in steatosis (OR 1.83; 95\% CI 1.03, 3.25; $\left.I^{2}=35 \%, p=0.04\right)$ and lobular inflammation (OR 1.57; $95 \%$ CI $\left.1.03,2.39 ; I^{2}=0 \%, p=0.04\right)$. One RCT reported also an improvement in NAS score with Viusid [98]. 
Antioxidants as a group or vitamin E did not slow fibrosis progression (Fig. 8).

Predictors of histological response to antioxidants are unclear: weight loss, circulating oxidative stress markers or vitamin E deficiency do not predict histological response $[49,95,96,100]$. There was no significant publication bias (ESM Fig. 1)

Glucose metabolism and cardiovascular risk Antioxidants had no effect on body weight, waist circumference, LDL-C and HDL-C. For HOMA, FPG and TG heterogeneity was high (Fig. 8): when considering only the RCTs with vitamin $\mathrm{E}$, active treatment had no significant effect on FPG (WMD $-0.04,95 \% \mathrm{CI}-0.66,0.57, p=0.89, I^{2}=0 \%, n$ comparisons $=$ $5)$, but was associated with a modestly higher risk of increasing HOMA (WMD 10.5, 95\% CI 0.3, 20.6, $p=0.04, \mathrm{I}^{2}=45 \%$, $n$ comparisons $=4)$ and plasma TG (WMD $6.00,95 \%$ CI 1.26 , 10.75, $p=0.01, I^{2}=0 \%, n$ comparisons $=4$ ) compared with controls. One RCT showed an improvement in plasma adiponectin with the combination of UDCA+vitamin E compared with placebo [88]. There was no significant publication bias for assessed outcomes (not reported).

Other drugs: L-carnitine, probiotics, incretin analogues, caspase inhibitors

L-carnitine is a precursor of carnitine-palmitoyltransferase, the rate-limiting enzyme in mitochondrial fatty acid transport and oxidation. When added to lifestyle intervention for 6 months, it improved steatosis, inflammation, fibrosis, HOMA, FPG, plasma lipids and C-reactive protein (ESM Table 5) [101].

Gut bacteria may promote liver injury and systemic inflammation through endotoxin-mediated toll-like receptor-4 axis activation and other mechanisms, predisposing to NASH, diabetes and atherosclerosis [102]. Three RCTs assessed probiotics in NAFLD: the first, evaluating VSL3, was prematurely terminated for an increase in hepatic steatosis [103]; the others, assessing a mixture of Lactobacillus spp. plus either Bifidobacterium bifidum or Streptococcus thermophilus, found a significant improvement in MRS-assessed hepatic fat and aminotransferases, respectively [104, 105].

The effect of probiotics in NAFLD is being evaluated in clinical trials (trial registration clinicaltrials.gov NCT00099723, NCT00808990, NCT00870012).

Incretin GLP-1 analogues improved insulin secretion by stimulating pancreatic beta cell growth and insulin release, and also improved hepatic steatosis and insulin resistance in mouse models [106]. Exenatide significantly improved transaminases in three RCTs enrolling diabetic patients [107], and its effects on liver histology in NASH are being tested in clinicaltrials.gov NCT00529204 and NCT00650546. In the Liraglutide Effect and Action in Diabetes (LEAD)-2 RCT,
2 years of liraglutide significantly reduced liver enzymes, CT-assessed hepatic steatosis, body fat and blood pressure and improved glycaemic control in diabetic patients with NAFLD (ESM Table 5) [108].

In NASH, hepatocyte apoptosis correlates with disease severity, and reducing hepatocyte apoptosis may has a potential for altering the course of the liver disease. In a phase 2 RCT, 124 patients with biopsy-proven NASH were randomised to once-daily placebo or $1,5,10$ or $40 \mathrm{mg}$ of the selective caspase inhibitor GS-9450 for 4 weeks: at EOT, NASH patients treated with 5-40 mg/day of GS-9450 significantly improved ALT levels, but not other metabolic variables, without significant side effects [109].

\section{Discussion}

Implications for practice

Weight loss is safe and may benefit both liver and cardio-metabolic disease in NAFLD: although a $\geq 5 \%$ weight loss improves steatosis and cardio-metabolic variables, $\mathrm{a} \geq 7 \%$ weight loss improves also histological disease activity in NASH; however, the latter goal was achieved by $<50 \%$ individuals even in RCTs adopting intensive multidisciplinary lifestyle interventions, making patient compliance a concern $[8,10]$.

Regular moderate-intensity aerobic exercise should be implemented in lifestyle intervention, as it enhances whole body lipid oxidation, and improves steatosis and cardio-metabolic risk profile regardless of weight loss: it may also protect NAFLD patients against the development of diabetes [110].

For patients with NASH not responding to lifestyle intervention, pharmacological treatment should be considered. Currently, no specific pharmacological treatment can be recommended outside clinical trials, for long-term safety and efficacy concerns. Among available agents, TZDs, statins, PUFA and antioxidants have been most extensively evaluated. Statins and PUFA ameliorate steatosis and liver enzymes, but their impact on liver histology is unknown,

TZDs improve steatosis and necroinflammation, slow fibrosis progression, and ameliorate glucose and lipid metabolism and subclinical inflammation, with more consistent cardiovascular benefits with pioglitazone. These findings should not be underestimated, given the key role of fibrosis in the progression of NAFLD to end-stage liver disease, and pioglitazone warrants evaluation in a larger RCT of longer duration. Open issues on TZDs are their long-term safety and efficacy, how to prevent their side effects and the development of predictors of histological response to these drugs. 
Antioxidants yielded mixed results on liver histology, improving histological disease activity when administered for 2 years or when implemented with vigorous weight-loss regimens [97].

Differently from TZDs, vitamin E worsened insulin resistance and plasma TG. Several studies found that vitamins E may preclude the insulin-sensitising effects of exercise by hampering physiological training-induced cellular adaptations in muscle in healthy individuals: vitamin E supplementation prevented exercise-induced production of PPAR $-\gamma$, PPAR- $\gamma$ coactivators PGC $1 \alpha$ and PGC1 $1 \beta$, and antioxidant enzymes superoxide dismutase and glutathione peroxidase [111]. Although these data have not been recently confirmed [112, 113], the impact of antioxidants on muscle insulin sensitivity in insulin-resistant individuals is unclear. An increased all-cause mortality has been associated with long-term administration of doses of vitamin E typically used in these trials [114]. Finally, antioxidant effectiveness in diabetic NAFLD patients, characterised by prominent systemic oxidative stress and severe liver histology, is unknown, as only $9 \%$ of enrolled patients were diabetic.

\section{Implications for future research}

With the exception of the GREACE trial [55], no RCT had adequate size and duration to evaluate clinical outcomes. Therefore, future RCTs need to assess if the observed benefits on intermediate endpoints will translate into a reduction of liver-related and cardio-metabolic morbidity and mortality.

The optimal dietary nutrient composition for NAFLD is an uncovered field: the role of excessive fructose, cholesterol and trans fat for NAFLD pathogenesis, as suggested by epidemiological and experimental studies, deserves evaluation in therapeutic RCTs. Fructose and high-fructose corn syrup, a common soft drink sweetener, in particular, have been independently connected to the risk and severity of NAFLD in population-based studies and in a randomised crossover trial [115-119].

The role of alcohol consumption in NAFLD needs also further evaluation: retrospective data suggest a protective role for light-to-moderate $(<10-20 \mathrm{~g} /$ day $)$ alcohol intake against insulin resistance, metabolic syndrome and NAFLD $[120,121]$. By contrast, modest alcohol intake and obesity seem to have additive effect on liver disease progression, and in a large prospective study any degree of alcohol consumption increased by 3.6-fold the risk of hepatocellular carcinoma in NASH-related cirrhosis [122, 123].

Cigarette smoking, an established risk factor for CVD and metabolic syndrome, has been epidemiologically linked to the onset and severity of NASH [124-126]. In the GREACE trial [54], current smokers had an OR of having baseline abnormal liver enzymes of $3.03(95 \%$
CI $1.99,4.64)$ compared with non-smokers. These data prompt evaluation of the effects of smoking cessation on NAFLD in future RCTs.

With the possible exceptions of telmisartan and pentoxifylline (limited evidence from two small RCTs), available agents do not improve hepatic fibrosis, the features most consistently associated with adverse liver-related outcomes. This may have several explanations: the slower progression rate $(0.1-0.2$ stages per year) of fibrosis [127] may require larger and longer RCTs to show fibrosis regression, and the encouraging results of TZDs on fibrosis progression are consistent with this view; alternatively, hepatic fibrogenesis may involve different molecular mechanisms from those involved in dysmetabolism, steatosis and inflammation. Within this context, antifibrotic agents targeting directly hepatic stellate cell activation and collagen deposition/remodelling, including toll-like receptor-4, tissue inhibitors of metalloproteinases (TIMPs) and FXR, are under development and phase III RCTs are eagerly awaited [128].

Our analysis provides also some hints for methodological improvement of future RCTs. Concerning cardio-metabolic risk of NAFLD, it is currently unclear whether NAFLD determines or is just a marker of associated cardio-metabolic abnormalities, and a comprehensive cardio-metabolic profiling of these patients may help predicting the impact of proposed treatments on cardio-metabolic outcomes [129]. As an example, $\mathrm{HbA}_{1 \mathrm{c}}$ (reported in only $22 \%$ of RCTs; Table 1 ) is emerging as a robust marker not only of recent glycaemic control in diabetes, but also of the risk of developing diabetes and CVD in diabetic and non-diabetic individuals [130,131].

The risk of developing or deteriorating type 2 diabetes is related to insulin resistance and pancreatic beta cell dysfunction [132]. In NAFLD, insulin resistance is universal, but impaired pancreatic beta cell function was also found in non-diabetic patients with NASH [133]. The different tissue insulin sensitivity also needs attention. Most RCTs adopted fasting insulin sensitivity indices (HOMA and QUICKI) (Table 1), which are easy to measure, predict incident CVD and diabetes in the general population and overall mortality in NAFLD [134, 135], but may have some limitations in such a complex disease as NAFLD. Insulin sensitivity is tissue-specific and skeletal muscle (i.e. the ability of insulin to enhance glucose disposal in muscle), hepatic (i.e.

Fig. 8 Forest plots of RCTs comparing the effect of antioxidants on liver disease, glucose metabolism and cardiovascular risk. (a) Improvement in histological steatosis in NASH. (b) Improvement in lobular inflammation in NASH. (c) Improvement in hepatocellular ballooning in NASH. (d) Improvement in fibrosis in NASH. (e) Improvement or stability in fibrosis in NASH. (f) Body weight change (\%). (g) Waist circumference change (\%). (h) HOMA index change (\%). (i) FPG change (\%). (j) Plasma LDL-cholesterol change (\%). (k) Plasma HDL-cholesterol change (\%). (l) Plasma TG change (\%).M-H, Mantel-Haenszel 
a

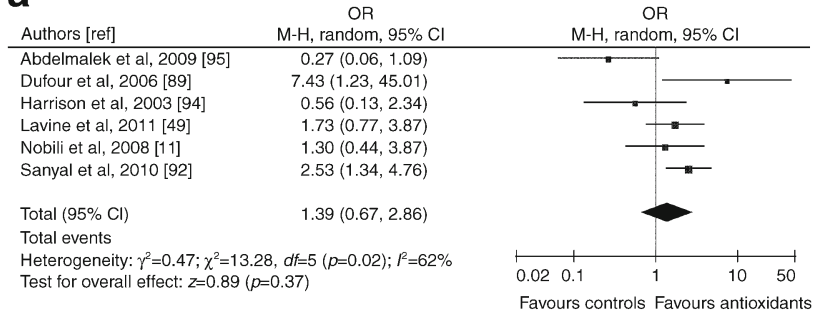

\section{C}

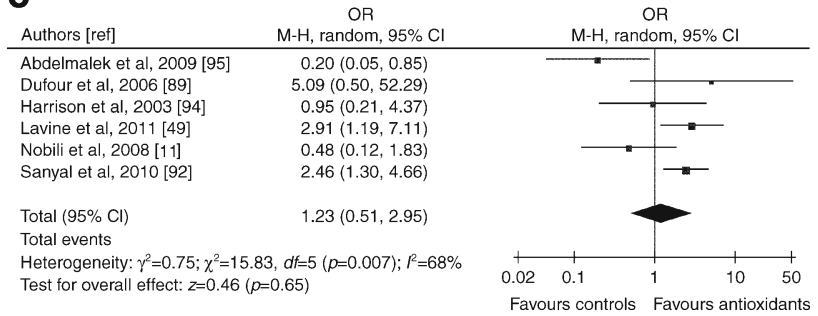

\section{e}

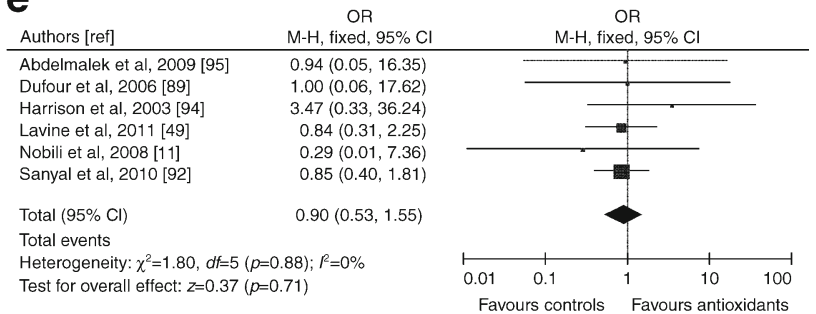

\section{g}

Authors [ref]

Lavine et al, 2011 [49]

Sanyal et al, 2010 [92]

Vilar Gomez et al, 2009 [98]

IV, fixed, $95 \% \mathrm{C}$

$0.00(-0.73,0.73)$

$-0.60(-2.42,1.22)$

Total $(95 \% \mathrm{Cl})$

$0.60(-0.92,2.12)$

$\chi^{2}=1.01, d f=2(p=0.60) ; R=0 \%$

Test for overall effect: $z=0.10(p=0.92)$

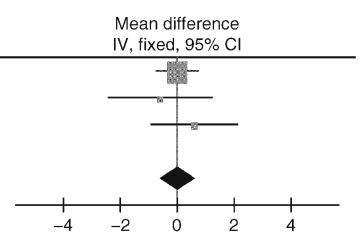

Favours antioxidants Favours control i

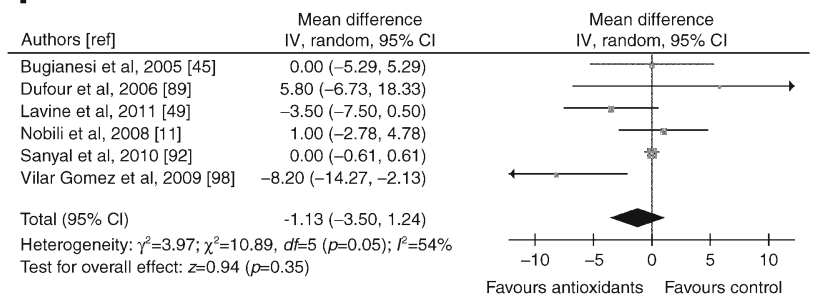

b

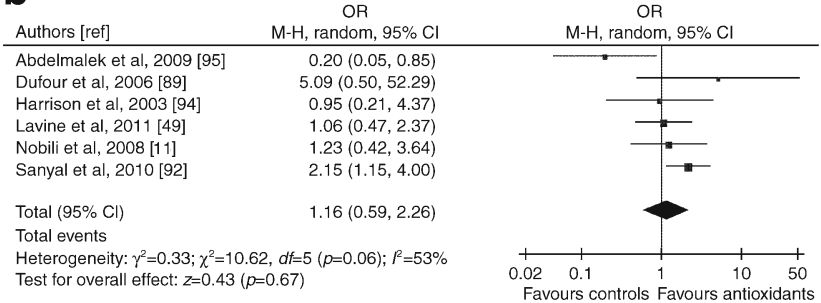

d

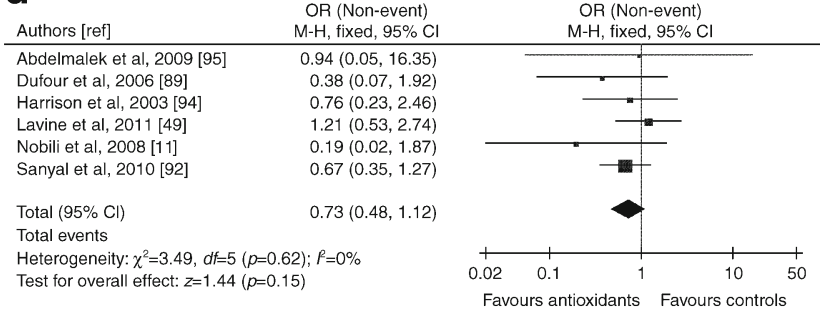

f

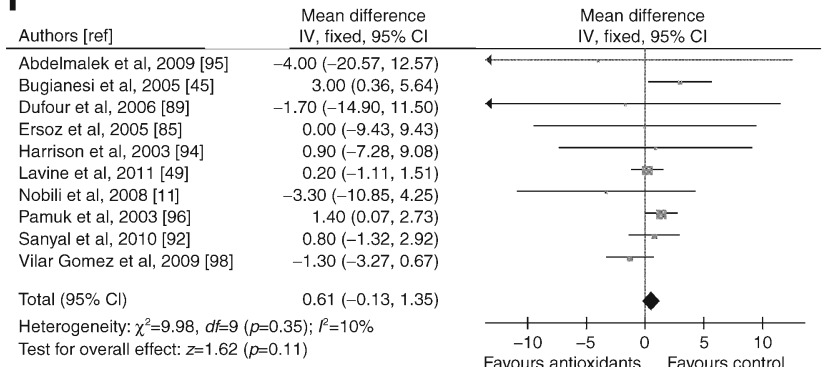

h

\begin{tabular}{|c|c|c|}
\hline Authors [ref] & $\begin{array}{l}\text { Mean difference } \\
\mathrm{IV} \text {, random, } 95 \% \mathrm{CI}\end{array}$ & $\begin{array}{l}\text { Mean difference } \\
\text { IV, random, } 95 \% \mathrm{CI}\end{array}$ \\
\hline Abdelmalek et al, 2009 [95] & $26.00(0.81,51.19)$ & \\
\hline Bugianesi et al, 2005 [45] & $2.40(-20.49,25.29)$ & \\
\hline Dufour et al, 2006 [89] & $-46.00(-85.16,-6.84)$ & \\
\hline Lavine et al, 2011 [49] & $20.00(6.51,33.49)$ & \\
\hline Nobili et al, 2008 [11] & $16.40(1.49,31.31)$ & \\
\hline Sanyal et al, 2010 [92] & $1.00(-10.77,12.77)$ & \\
\hline Vilar Gomez et al, 2009 [98] & $-6.30(-19.20,6.60)$ & \\
\hline Total $(95 \% \mathrm{Cl})$ & $5.49(-6.27,17.25)$ & \\
\hline $\begin{array}{l}\text { Heterogeneity: } \gamma^{2}=159.11 ; \chi^{2} \\
\text { Test for overall effect: } z=0.91\end{array}$ & $\begin{array}{l}\text { P.48, } d f=6(p=0.003) ; i^{2}=69 \% \\
=0.36)\end{array}$ & $\begin{array}{lllll}-20 & -10 & 0 & 10 & 20 \\
\text { antioxidants } & \text { Favours controls }\end{array}$ \\
\hline
\end{tabular}

j

\begin{tabular}{|c|c|c|}
\hline Authors [ref] & $\begin{array}{l}\text { Mean difference } \\
\text { IV, fixed, } 95 \% \mathrm{Cl}\end{array}$ & $\begin{array}{l}\text { Mean difference } \\
\text { IV, fixed, } 95 \% \mathrm{Cl}\end{array}$ \\
\hline Lavine et al, 2011 [49] & $1.10(-6.91,9.11)$ & 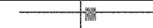 \\
\hline Sanyal et al, 2010 [92] & $-5.50(-11.87,0.87)$ & -1 \\
\hline Total $(95 \% \mathrm{Cl})$ & $-2.94(-7.93,2.04)$ & \\
\hline Heterogeneity: $\chi^{2}=1.60$ & $21) ; P^{2}=37 \%$ & 5 \\
\hline
\end{tabular}

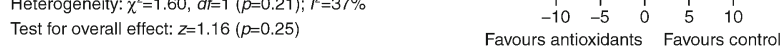

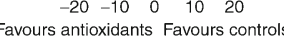

\section{k}

\begin{tabular}{lr} 
Authors [ref] & $\begin{array}{r}\text { Mean difference } \\
\text { IV, fixed, } 95 \% \mathrm{Cl}\end{array}$ \\
\hline Lavine et al, 2011 [49] & $-3.20(-9.02,2.62)$ \\
Sanyal et al, 2010 [92] & $2.40(-4.27,9.07)$ \\
& $-0.78(-5.17,3.61)$ \\
Total $(95 \%$ Cl) & $\begin{array}{l}\text { Heterogeneity: } \chi^{2}=1.54, d f=1 \quad(p=0.22) ; P=35 \% \\
\text { Test for overall effect: } z=0.35(p=0.73)\end{array}$
\end{tabular}

Mean difference

Favours antioxidants $\begin{array}{ccr}0 & 5 & 10 \\ & & \text { Favours control }\end{array}$

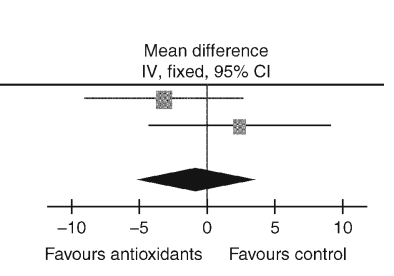

\begin{tabular}{lc} 
Authors [ref] & $\begin{array}{c}\text { Mean difference } \\
\text { IV, random, 95\% Cl }\end{array}$ \\
\hline Lavine et al, 2011 [49] & $10.30(-8.99,29.59)$ \\
Nobili et al, 2008 [11] & $7.40(-17.95,32.75)$ \\
Pamuk et al, 2003 [96] & $6.50(0.53,12.47)$ \\
Sanyal et al, 2010 [92] & $3.70(-5.40,12.80)$ \\
Vilar Gomez et al, 2009 [98] & $37.20(-55.92,-18.48)$ \\
& \\
Total (95\% Cl) & $-1.14(-13.96,11.67)$ \\
Heterogeneity: $\gamma^{2}=150.67 ; \chi^{2}=19.69, d f=4(p=0.0006) ; l^{2}=80 \%$ \\
Test for overall effect: $z=0.17(p=0.86)$
\end{tabular}

Heterogeneity: $\gamma^{2}=150.67 ; \chi^{2}=19.69$,
Test for overall effect: $z=0.17(p=0.86)$

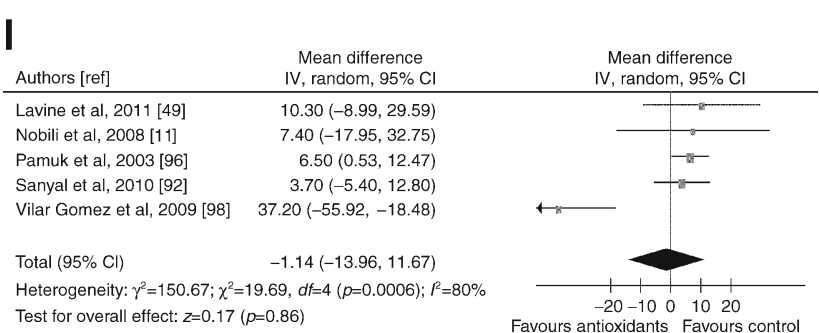


the ability of insulin to suppress hepatic glucose output in fasting conditions) and adipose tissue (i.e. the ability of insulin to suppress adipose tissue lipolysis) insulin sensitivity do not always parallel each other and may differently relate to liver and cardio-metabolic disease: whereas liver injury seems tightly related to adipose tissue insulin sensitivity in NASH [26], hepatic or muscle insulin sensitivity are more tightly related to glucose tolerance and the risk of future diabetes [136]. This may explain why metformin does not affect liver histology despite constant HOMA reduction and, similarly, the lack of improvement in liver injury despite continued HOMA improvement observed in the FLIRT trials. Therefore, different tissue insulin sensitivity should be systematically assessed, together with pancreatic beta cell function, with a simple standard OGTT, without applying the more troublesome glucose clamp technique [137].

Plasma inflammatory markers are also emerging as important tools in risk assessment and targeting of therapy in patients with metabolic syndrome and could be extended to RCTs on NAFLD [138].

In conclusion, weight loss and pioglitazone seem to most extensively benefit intermediate endpoints in NAFLD, improving not only liver disease but also cardiometabolic variables [139], while vitamin E improves histological disease activity but may worsen the cardiometabolic profile. The latter issue, as well as the risk/ benefit profile of other antioxidants in NAFLD [140], needs further evaluation in future RCTs adequately powered for clinical outcomes.

Acknowledgements The authors are indebted to the following colleagues, who provided additional data on randomised controlled trials: K. Cusi, University of Florida at Gainesville, FL, USA; J.E. Lavine, Columbia University Medical Center, New York, USA; R. Loomba, Division of Gastroenterology, University of California at San Diego, USA; M.L. van Natta, Department of Epidemiology, Columbia University Medical Center, New York, USA.

Funding This work was supported in part by the Piedmont Region Funds Comitato Interministeriale per la Programmazione Economica 2008, which were employed for data collection. No funding bodies played any role in the design, writing or decision to publish this manuscript.

Duality of interest The authors declare that there is no duality of interest associated with this manuscript.

Contribution statement GM conceived and designed the study, analysed data, discussed results, wrote the manuscript and approved the final version of the manuscript. MC, FR and RG analysed data, revised the manuscript critically for important intellectual content and approved the final version of the manuscript.

\section{References}

1. Ratziu V, Bellentani S, Cortez-Pinto H, Day C, Marchesini G (2010) A position statement on NAFLD/NASH based on the EASL 2009 special conference. J Hepatol 53:372-384

2. Younossi ZM, Stepanova M, Afendy M et al (2011) Changes in the prevalence of the most common causes of chronic liver diseases in the United States from 1988 to 2008. Clin Gastroenterol Hepatol 9:524-530

3. Musso G, Gambino R, Cassader M, Pagano G (2011) Prognosis and non-invasive methods to assess liver disease severity in non-alcoholic fatty liver disease (NAFLD): systematic review and meta-analysis. Ann Med 43:617-649

4. Ghouri N, Preiss D, Sattar N (2010) Liver enzymes, nonalcoholic fatty liver disease, and incident cardiovascular disease: a narrative review and clinical perspective of prospective data. Hepatology 52:1156-1161

5. Higgins JPT, Green S (eds) (2008) Cochrane Handbook for Systematic Reviews of Interventions, Version 5.0.0. The Cochrane Collaboration. Available at www.Cochranehandbook.org. Accessed 26 May 2009

6. Kugelmas M, Hill DB, Vivian B, Marsano L, McClain CJ (2003) Cytokines and NASH: a pilot study of the effects of lifestyle modification and vitamin E. Hepatology 38:413-419

7. Zelber-Sagi S, Kessler A, Brazowsky E et al (2006) A double-blind randomized placebo-controlled trial of orlistat for the treatment of non-alcoholic fatty liver disease. Clin Gastroenterol Hepatol 4:639-644

8. Lazo M, Solga SF, Horska A et al (2010) The effect of a 12 -month intensive lifestyle intervention on hepatic steatosis in adults with type 2 diabetes. Diabetes Care 33:2156-2163

9. Shah K, Stufflebaum A, Hilton TN et al (2009) Diet and exercise intervention reduce intrahepatic fat content and improve insulin sensitivity in obese adult older adults. Obesity 17:2162-2168

10. Promrat K, Kleiner DE, Niemeier HM et al (2010) Randomized controlled trial testing the effects of weight loss on nonalcoholic steatohepatitis. Hepatology 51:121-129

11. Nobili V, Manco M, Devito R et al (2008) Lifestyle intervention and antioxidant therapy in children with nonalcoholic fatty liver disease: a randomized, controlled trial. Hepatology 48: $119-128$

12. Harrison SA, Brunt EM, Fecht WJ, Neuschwander-Tetri BA (2009) Orlistat for overweight subjects with nonalcoholic steatohepatitis (NASH): a randomized prospective trial. Hepatology 49:80-86

13. Hayward CS, Lockwood J, Williams CD, Cole RE, Torres DM, Harrison SA (2010) Lifestyle modification and NAFLD: a prospective randomized trial. Hepatology 52(S4):622A

14. Kistler K, Brunt EM, Clark JM et al (2011) Physical activity recommendations, exercise intensity, and histological severity of nonalcoholic fatty liver disease. Am J Gastroenterol 106:460-468

15. Perseghin G, Lattuada G, de Cobelli F et al (2007) Habitual physical activity is associated with intrahepatic fat content in humans. Diabetes Care 30:683-688

16. Zelber-Sagi S, Nitzan-Kaluski D, Goldsmith R et al (2008) Role of leisure-time physical activity in non-alcoholic fatty liver disease; a population-based study. Hepatology 48:17911798

17. Rabøl R, Falk Petersen K, Dufour S, Flannery C S, Shulman GL (2011) Reversal of muscle insulin resistance with exercise reduces postprandial hepatic de novo lipogenesis in insulin resistant individuals. Proc Natl Acad Sci U S A 108:13705-13709

18. Bonekamp S, Barone BB, Clark J, Stewart KJ (2008) The effects of an exercise training intervention on hepatic steatosis. Hepatology 48(Suppl 1):806A 
19. Johnson NA, Sachinwalla T, Walton DW et al (2009) Aerobic exercise training reduces hepatic and visceral lipids in obese individuals without weight loss. Hepatology 50:1105-1112

20. St. George A, Bauman A, Johnston A et al (2009) Independent effects of physical activity in patients with non-alcoholic fatty liver. Hepatology 50:68-76

21. Hallsworth K, Fattakhowa G, Hollingsworth K et al (2011) Resistance exercise reduces liver fat and its mediators in non-alcoholic fatty liver disease independent of weight loss. Gut 60:1278-1283

22. Frith J, Day CP, Robinson L, Elliott C, Jones DE, Newton JL (2010) Potential strategies to improve uptake of exercise interventions in non-alcoholic fatty liver disease. J Hepatol 52:112-116

23. Kirk E, Reeds DN, Finck BN, Mayurranjan MS, Klein S (2009) Dietary fat and carbohydrates differentially alter insulin sensitivity during caloric restriction. Gastroenterology 136:1552-1560

24. Haufe S, Engeli S, Kast P et al (2011) Randomized comparison of reduced fat and reduced carbohydrate hypocaloric diets on intrahepatic fat in overweight and obese human subjects. Hepatology 53:1504-1514

25. de Luis DA, Aller R, Izaola O, Gonzalez Sagrato M, Conde R (2010) Effect of two different hypocaloric diets in transaminases and insulin resistance in nonalcoholic fatty liver disease and obese patients. Nutr Hosp 25:730-735

26. Gastaldelli A, Harrison SA, Belfort-Aguilar R et al (2009) Importance of changes in adipose tissue insulin resistance to histological response during thiazolidinedione treatment of patients with nonalcoholic steatohepatitis. Hepatology 50:1087-1093

27. Sanyal AJ, Mofrad PS, Contos MJ et al (2004) A pilot study of vitamin $\mathrm{E}$ versus vitamin $\mathrm{E}$ and pioglitazone for the treatment of nonalcoholic steatohepatitis. Clin Gastroenterol Hepatol 2:1107-1115

28. Belfort R, Harrison SA, Brown K et al (2006) A placebocontrolled trial of pioglitazone in subjects with nonalcoholic steatohepatitis. N Engl J Med 355:2297-2307

29. Aithal GP, Thomas JA, Kaye PV et al (2008) Randomized, placebo-controlled trial of pioglitazone in nondiabetic subjects with nonalcoholic steatohepatitis. Gastroenterology 135:11761184

30. Ratziu V, Giral P, Jacqueminet S et al (2008) Rosiglitazone for nonalcoholic steatohepatitis: one-year results of the randomized placebo-controlled Fatty Liver Improvement with Rosiglitazone Therapy (FLIRT) Trial. Gastroenterology 135:100-110

31. Idilman R, Mizrak D, Corapcioglu D et al (2008) Clinical trial: insulin-sensitizing agents may reduce consequences of insulin resistance in individuals with non-alcoholic steatohepatitis. Aliment Pharmacol Ther 28:200-208

32. Sanyal AJ, Chalasani N, Kowdley KV et al (2010) Pioglitazone, vitamin $\mathrm{E}$, or placebo for nonalcoholic steatohepatitis. $\mathrm{N}$ Engl $\mathrm{J}$ Med 362:1675-1685

33. Omer Z, Cetinkalp S, Akyildiz M et al (2010) Efficacy of insulinsensitizing agents in nonalcoholic fatty liver disease. Eur $\mathrm{J}$ Gastroenterol Hepatol 22:18-23

34. Tiikkainen M, Häkkinen AM, Korsheninnikova E, Nyman T, Mäkimattila S, Yki-Järvinen H (2004) Effects of rosiglitazone and metformin on liver fat content, hepatic insulin resistance, insulin clearance, and gene expression in adipose tissue in patients with type 2 diabetes. Diabetes 53:2169-2173

35. Jonker JT, Wang Y, de Haan W et al (2010) Pioglitazone decreases plasma cetp mass, associated with a decrease in hepatic triglyceride content, in patients with type 2 diabetes mellitus. Diabetes Care 33:1625-1628

36. Shah PK, Mudaliar S, Chang AR et al (2011) Effects of intensive insulin therapy alone and in combination with pioglitazone on body weight, composition, distribution and liver fat content in patients with type 2 diabetes. Diabetes Obes Metabol 13:505-510
37. Gupta AK, Bray GA, Greenway FL et al (2010) Pioglitazone, but not metformin, reduces liver fat in type- 2 diabetes mellitus independent of weight changes. J Diabetes Complications 24:289-296

38. Torres D, Jones F, Shaw J, Williams C, Ward J, Harrison S (2011) Rosiglitazone versus rosiglitazone and metformin versus rosiglitazone and losartan in the treatment of nonalcoholic steatohepatitis (NASH): a 12 month-randomized, prospective, open- label trial. Hepatology. doi:10.1002/hep.24558

39. Lutchamn G, Modi A, Kleiner DE et al (2007) The effects of discontinuing pioglitazone in patients with non-alcoholic steatohepatitis. Hepatology 46:424-429

40. Ratziu V, Charlotte F, Bernhardt C et al (2010) Long-term efficacy of rosiglitazone in nonalcoholic steatohepatitis: results of the FLIRT-2 extension trial. Hepatology 51:445-453

41. Zhou G, Myers R, Li Y et al (2001) Role of AMP-activated protein kinase in mechanisms of metformin action. J Clin Invest 108:1167-1174

42. Uygun A, Kadayifci A, Isik AT et al (2004) Metformin in the treatment of patients with non-alcoholic steatohepatitis. Aliment Pharmacol Ther 19:537-544

43. Haukeland JW, Konopski Z, Beate Eggesbø H et al (2009) Metformin in patients with non-alcoholic fatty liver disease: a randomized, controlled trial. Scand J Gastroenterol 44:853-860

44. Shields WW, Thompson KE, Grice GA, Harrison SA, Coyle WJ (2009) The effect of metformin and standard therapy versus standard therapy alone in nondiabetic patients with insulin resistance and nonalcoholic steatohepatitis (NASH): a pilot trial. Ther Adv Gastroenterology 2:157-163

45. Bugianesi E, Gentilcore E, Manini R et al (2005) A randomized controlled trial of metformin versus vitamin $\mathrm{E}$ or prescriptive diet in nonalcoholic fatty liver disease. Am J Gastroenterol 100: 1082-1090

46. Nar A, Gedik O (2009) The effect of metformin on leptin in obese patients with type 2 diabetes mellitus and nonalcoholic fatty liver disease. Acta Diabetol 46:113-118

47. Garinis GA, Fruci B, Mazza A et al (2010) Metformin versus dietary treatment in nonalcoholic hepatic steatosis: a randomized study. Int J Obes (Lond). doi:10.1038/ijo.2010.40

48. Tock L, Dâmaso AR, de Piano A et al (2010) Long-term effects of metformin and lifestyle modification on nonalcoholic fatty liver disease obese adolescents. J Obes. doi:10.1155/2010/831901

49. Lavine JE, Schwimmer JB, van Natta ML et al (2011) Effect of vitamin $\mathrm{E}$ or metformin for treatment of nonalcoholic fatty liver disease in children and adolescents: the TONIC randomized controlled trial. JAMA 305:1659-1668

50. Sofer E, Boaz M, Matas Z, Mashavi M, Shargorodsky M (2011) Treatment with insulin sensitizer metformin improves arterial properties, metabolic parameters, and liver function in patients with nonalcoholic fatty liver disease: a randomized, placebocontrolled trial. Metabolism 60:1278-1284

51. Cohen DE, Anania FA, Chalasani N (2006) An assessment of statin safety by hepatologists. Am J Cardiol 97(8A):77C-81C

52. Sattar N, Preiss D, Murray HM et al (2010) Statins and risk of incident diabetes: a collaborative meta-analysis of randomised statin trials. Lancet 375:735-742

53. Athyros VG, Mikhailidis DP, Didangelos TP et al (2006) Effect of multifactorial treatment on non-alcoholic fatty liver disease in metabolic syndrome: a randomised study. Curr Med Res Opin 22:873-883

54. Foster T, Budoff MJ, Saab S, Ahmadi N, Gordon C, Guerci AD (2011) Atorvastatin and antioxidants for the treatment of nonalcoholic fatty liver disease: the St Francis Heart Study randomized clinical trial. Am J Gastroenterol 106:71-77

55. Athyros VG, Tziomalos K, Gossios TD et al (2010) Safety and efficacy of long-term statin treatment for cardiovascular events in 
patients with coronary heart disease and abnormal liver tests in the Greek Atorvastatin and Coronary Heart Disease Evaluation (GREACE) Study: a post-hoc analysis. Lancet 376:1916-1922

56. Nelson A, Torres DM, Morgan AE, Fincke C, Harrison SA (2009) A pilot study using simvastatin in the treatment of nonalcoholic steatohepatitis: a randomized placebo-controlled trial. J Clin Gastroenterol 43:990-994

57. Wouters K, van Gorp PJ, Bieghs V et al (2008) Dietary cholesterol, rather than liver steatosis, leads to hepatic inflammation in hyperlipidemic mouse models of NASH. Hepatology 48: 474-486

58. Yasutake K, Nakamuta M, Shima Y et al (2009) Nutritional investigation of non-obese patients with non-alcoholic fatty liver disease: the significance of dietary cholesterol. Scand J Gastroenterol 44:471-477

59. Enjoji M, Nakamuta M (2010) Is the control of dietary cholesterol intake sufficiently effective to ameliorate nonalcoholic fatty liver disease? World J Gastroenterol 16:800-803

60. Zheng S, Hoos L, Cook J et al (2008) Ezetimibe improves high fat and cholesterol diet-induced non-alcoholic fatty liver disease in mice. Eur J Pharmacol 584:118-124

61. Nozaki Y, Fujita K, Yoneda M et al (2009) Long-term combination therapy of ezetimibe and acarbose for non-alcoholic fatty liver disease. J Hepatol 51:548-556

62. Deushi M, Nomura M, Kawakami A et al (2007) Ezetimibe improves liver steatosis and insulin resistance in obese rat model of metabolic syndrome. FEBS Lett 581:5664-5670

63. Chan DC, Watts GF, Gan SK, Ooi EM (2010) Barrett PH Effect of ezetimibe on hepatic fat, inflammatory markers and apolipoprotein B-100 kinetics in insulin-resistant obese subjects on a weight loss diet. Diabetes Care 33:1134-1139

64. Takeshita Y, Takamura T, Kita Y et al (2011) Efficacy of ezetimibe for the treatment of non-alcoholic fatty liver disease: a randomized controlled trial. J Hep 54:S346

65. Nobili V, Bedogni G, Alisi A et al (2011) Docosahexaenoic acid supplementation decreases liver fat content in children with non-alcoholic fatty liver disease: double-blind randomised controlled clinical trial. Arch Dis Child 96:350-353

66. Spadaro L, Magliocco O, Spampinato D et al (2008) Effects of n-3 polyunsaturated fatty acids in subjects with nonalcoholic fatty liver disease. Dig Liv Dis 40:194-199

67. Zhu FS, Liu S, Chen XM, Huang ZG, Zhang DW (2008) Effects of n-3 polyunsaturated fatty acids from seal oils on nonalcoholic fatty liver disease associated with hyperlipidemia. World J Gastroenterol 14:6395-6400

68. Cussons AJ, Watts GF, Mori TA, Stuckey BGA (2009) Omega-3 fatty acid supplementation decreases liver fat content in polycystic ovary syndrome: a randomized controlled trial employing proton magnetic resonance spectroscopy. J Clin Endocrinol Metab 94:3842-3848

69. Caldwell SH, Argo CK, Henry TD et al (2011) Dissociated histological and metabolic effects of omega-3 $(3,000 \mathrm{mg} / \mathrm{d}) \mathrm{vs}$. placebo with both exercise and diet in a double-blind randomized controlled trial of NASH. J Hep 54:S8

70. Merat S, Malekzadeh R, Sohrabi MR et al (2003) Probucol in the treatment of non-alcoholic steatohepatitis: a double-blind randomized controlled study. J Hepatology 38:414-418

71. Seo YS, Kim JH, Jo NY et al (2008) PPAR agonists treatment is effective in a nonalcoholic fatty liver disease animal model by modulating fatty-acid metabolic enzymes. J Gastroenterol Hepatol 23:102-109

72. Basaranoglu M, Acbay O, Sonsuz A (1999) A controlled trial of gemfibrozil in the treatment of patients with nonalcoholic steatohepatitis. J Hepatol 31:384

73. Conjeevaram HS, McKenna BJ, Kang H et al (2009) A randomized placebo-controlled study of PPAR-alpha agonist fenofibrate in patients with nonalcoholic steatohepatitis (NASH). Hepatology 50 (S4):774A

74. Korenblat K, Fabbrini E, Mohammed BS et al (2009) Effects of fenofibrate and long-acting nicotinic acid on intrahepatic triglyceride content and adipose tissue insulin sensitivity in obese human subjects. J Hep 50:S25

75. Fabbrini E, Mohammed BS, Korenblat KM et al (2010) Effect of fenofibrate and niacin on intrahepatic triglyceride content, very low-density lipoprotein kinetics, and insulin action in obese subjects with nonalcoholic fatty liver disease. J Clin Endocrinol Metab 95:2727-2735

76. Georgescu EF, Ionescu R, Georgescu M et al (2009) Angiotensinreceptor blockers as therapy for mild-to-moderate hypertensionassociated non-alcoholic steatohepatitis. World J Gastroenterol 15:942-954

77. Fogari R, Mugellini A, Zoppi A et al (2011) Losartan alone or in combination with simvastatin improved visceral adipose tissue and inflammation in hypertensive normocholesterolemic patients with nonalcoholic hepatic steatosis. Eur J Gastroenterol Hepatol. doi:10.1097/MEG.0b013e32834ba188

78. Després JP, Ross R, Boka G, Alméras N, Lemieux I (2009) Effect of rimonabant on the high-triglyceride/ low-HDL-cholesterol dyslipidemia, intraabdominal adiposity, and liver fat. The ADAGIO-Lipids Trial. Arterioscler Thromb Vasc Biol 29: 416-423

79. Buranawui W, Thung-u-thaisri P, Pramoolsinsap C et al (2010) Pentoxifylline for treatment of non-alcoholic fatty liver disease (NAFLD): a randomized, placebo-controlled study. Gastroenterology A330:s2161

80. Lee YM, Sutedja D, Wai CT et al (2008) A randomized controlled pilot study of pentoxifylline in patients with non-alcoholic steatohepatitis (NASH). Hepatol Int 2:196-201

81. Rinella ME, Koppe S, Brunt EM, Elias M, Gottstein J, Green RM (2010) Pentoxifylline improves ALT and histology in patients with NASH: a double-blind placebo controlled trial. Gastroenterology 136 (Supplement) Digestive Disease Week (CD-ROM)

82. Zein CO, Yerian LM, Gogate P et al (2011) Pentoxifylline improves nonalcoholic steatohepatitis: a randomized placebocontrolled trial. Hepatology. doi:10.1002/hep.24544

83. Santos VN, Lanzoni VP, Szejnfeld D, Shigueoka D, Parise ER (2003) A randomized double-blind study of the short-time treatment of obese patients with non-alcoholic fatty liver disease with ursodeoxycholic acid. Braz J Med Biol Res 36:723-729

84. Mendez-Sanchez N, Gonzalez V, Chavez-Tapia N, Ramos M, Uribe M (2004) Weight reduction and ursodeoxycholic acid in subjects with non-alcoholic fatty liver disease. A double-blind, placebo-controlled trial. Ann Hepatol 3:108-112

85. Ersoz G, Gunsar F, Karasu Z, Akay S, Batur Y, Akarca US (2005) Management of fatty liver disease with vitamin $\mathrm{E}$ and $\mathrm{C}$ compared to ursodeocycholic acid treatment. Turk J Gastroenterol $16: 124-128$

86. Ratziu V, de Ledinghen V, Oberti F et al (2011) A randomized controlled trial of high-dose ursodesoxycholic acid for nonalcoholic steatohepatitis. J Hepatol 54:1011-1019

87. Leuschner UFH, Lindenthal B, Herrmann G et al (2010) High-dose ursodeoxycholic acid therapy for nonalcoholic steatohepatitis: a double-blind, randomized, placebo-controlled trial. Hepatology 52:472-479

88. Lindor KD, Kowdley KV, Heathcote EJ et al (2004) Ursodeoxycholic acid for the treatment of non-alcoholic steatohepatitis: results of a randomized trial. Hepatology 39:770-778

89. Dufour JF, Oneta CM, Gonvers JJ et al (2006) Randomized placebo-controlled trial of ursodeoxycholic acid with vitamin $\mathrm{E}$ in non-alcoholic steatohepatitis. Clin Gastroenterol Hepatol 4:1537-1543 
90. Musso G, Gambino R, Cassader M (2010) Emerging molecular targets for the treatment of nonalcoholic fatty liver disease. Ann Rev Med 61:375-392

91. Knop FK (2010) Bile-induced secretion of glucagon-like peptide-1: pathophysiological implications in type 2 diabetes? Am J Physiol Endocrinol Metab 299:E10-E13

92. Sanyal AJ, Mudaliar S, Henry RR et al (2010) A new therapy for non-alcoholic fatty liver disease and diabetes? Int-747-the first FXR hepatic therapeutic study. Hepatology 50:389A

93. Rui M, Wang C, Fang J et al (2001) The clinical comparison of reduced glutathione and DaiNingPlan in the treatment of nonalcoholic steatohepatitis. Chin Gen Pract 4:269-270

94. Harrison SA, Torgerson S, Ward J et al (2003) Vitamin E and vitamin $\mathrm{C}$ treatment improves fibrosis in patients with nonalcoholic steatohepatitis. Am J Gastroenterol 98:2485-2490

95. Abdelmalek MF, Sanderson SO, Angulo P et al (2009) Betaine for nonalcoholic fatty liver disease: results of a randomized placebo-controlled trial. Hepatology 50:1818-1826

96. Pamuk GE, Sonsuz A (2003) $N$-acetylcysteine in the treatment of nonalcoholic steatohepatitis. J Gastroenterol Hepatol 18:1220-1221

97. Miglio F, Rovati LC, Santoro A, Senikar I (2000) Efficacy and safety of oral betaine glucuronate in non-alcoholic steatohepatitis. A double-blind, randomized, parallel group, placebo-controlled prospective clinical study. Arzneimittelforschung 50:722-727

98. Vilar Gomez E, Rodriguez de Miranda A, Gra Oramas B et al (2009) Clinical trial: Viusid(R) in combination with diet and exercise in patients with nonalcoholic fatty liver disease. Aliment Pharmacol Ther 30:999-1009

99. Loguercio C, Federico A, Trappoliere M et al (2007) The effect of a silybin-vitamin E-phospholipid complex on nonalcoholic fatty liver disease. Dig Dis Sci 52:2387-2395

100. Hashemi SJ, Hajiani E, Sardabi EH (2009) A placebo-controlled trial of silymarin in patients with nonalcoholic fatty liver disease. Hepat Mon 9:265-270

101. Neuschwander-Tetri BA, Sanyal AJ, Chalasani NP et al (2010) Changes in ALT and vitamin E levels and histological response in patients with NASH treated with vitamin E in the PIVENS trial. Hepatology 52:620A

102. Malaguarnera M, Gargante MP, Russo C et al (2010) L-Carnitine supplementation to diet: a new tool in treatment of nonalcoholic steatohepatitis. A randomized and controlled clinical trial. Am J Gastroenterol. doi:10.1038/ajg.2009.719

103. Musso G, Gambino R, Cassader M (2011) Interactions between gut microbiota and host metabolism predisposing to obesity and diabetes. Ann Rev Med 62:361-380

104. Solga SF, Buckley G, Clark JM, Horska A, Diehl AM (2008) The effect of a probiotic on hepatic steatosis. J Clin Gastroenterol 42:1117-1119

105. Wong VWS, Wong GLH, Wong CH, Chan HLY (2011) Treatment of non-alcoholic steatohepatitis with probioticsa proof-of-concept study with serial gut microbiota analysis by ultra-deep sequencing. J Hepatol 54:349

106. Aller R, de Luis DA, Izaola O et al (2011) Effect of a probiotic on liver aminotransferases in nonalcoholic fatty liver disease patients: a double blind randomized clinical trial. Eur Rev Med Pharmacol Sci 15:1090-1095

107. Zing X, Saxena NK, Lin S et al (2006) Exedin-4, a glucagon-like protein1 (GLP-1) receptor agonist, reverses hepatic steatosis in ob/ob mice. Hepatology 43:173-181

108. Buse JB, Klonoff DC, Nielsen LL (2007) Metabolic effects of two years of exenatide treatment on diabetes, obesity, and hepatic biomarkers in patients with type 2 diabetes: an interim analysis of data from the open-label, uncontrolled extension of three double-blind, placebo-controlled trials. Clin Ther 29:139-153
109. Jendle J, Nauck MA, Matthews DR et al (2009) Weight loss with liraglutide, a once-daily human glucagon-like peptide-1 analogue for type 2 diabetes treatment as monotherapy or added to metformin, is primarily as a result of a reduction in fat tissue. Diabetes Obes Metab 11:1163-1172

110. Ratziu V, Sheikh MY, Sanyal AJ et al (2011) A phase 2, randomized, double-blind, placebo-controlled study of GS-9450 in subjects with nonalcoholic steatohepatitis. Hepatology. doi:10.1002/hep. 24747

111. Arase Y, Suzuki F, Ikeda K et al (2009) Multivariate analysis of risk factors for the development of type 2 diabetes in nonalcoholic fatty liver disease. J Gastroenterol 44:1064-1070

112. Ristow M, Zarse K, Oberbach A et al (2009) Antioxidants prevent health-promoting effects of physical exercise in humans. Proc Natl Acad Sci U S A 106:8665-8670

113. Yfanti C, Akerström T, Nielsen S et al (2010) Antioxidant supplementation does not alter endurance training adaptation. Med Sci Sports Exerc 42:1388-1395

114. Yfanti C, Nielsen AR, Akerström T et al (2011) Effect of antioxidant supplementation on insulin sensitivity in response to endurance exercise training. Am J Physiol Endocrinol Metab 300:E761-E770

115. Miller ER, Pastor-Barriuso R et al (2005) Meta-analysis: high-dosage vitamin $\mathrm{E}$ supplementation may increase all-cause mortality. Ann Intern Med 142:37-46

116. Le KA, Ith M, Kreis R et al (2009) Fructose overconsumption causes dyslipidemia and ectopic lipid deposition in healthy subjects with and without a family history of type 2 diabetes. Am J Clin Nutr 89:1760-1765

117. Zelber-Sagi S, Nitzan-Kaluski D, Goldsmith R et al (2007) Long term nutritional intake and the risk for nonalcoholic fatty liver disease (NAFLD): a population based study. J Hepatol 47:711-717

118. Assy N, Nasser G, Kamayse I et al (2008) Soft drink consumption linked with fatty liver in the absence of traditional risk factors. Can J Gastroenterol 22:811-816

119. Ouyang X, Cirillo P, Sautin Y et al (2008) Fructose consumption as a risk factor for nonalcoholic fatty liver disease. J Hepatol 48:993-999

120. Abdelmalek MF, Suzuki A, Guy C et al (2010) Increased fructose consumption is associated with fibrosis severity in patients with NAFLD. Hepatology 51:1961-1971

121. Dunn W, Xu R, Schimmer JB (2008) Modest wine drinking and decreased prevalence of suspected non-alcoholic fatty liver disease. Hepatology 47:1947-1954

122. Cotrim HP, Freitas LA, Alves E et al (2009) Effects of lightto-moderate alcohol consumption on steatosis and steatohepatitis in severely obese patients. Eur J Gastroenterol Hepatol 21:969-972

123. Ruhl CE, Everhart JE (2005) Joint effects of body weight and alcohol on elevated serum alanine aminotransferase in the United States population. Clin Gastroenterol Hepatol 3:1260-1268

124. Ascha MS, Hanouneh IA, Lopez R, Tamimi TA, Feldstein AF, Zein NN (2010) The incidence and risk factors of hepatocellular carcinoma in patients with nonalcoholic steatohepatitis. Hepatology 51:1972-1978

125. Hamabe A, Uto H, Imamura $Y$ et al (2011) Impact of cigarette smoking on onset of nonalcoholic fatty liver disease over a 10 year period. J Gastroenterol 46:769-778

126. Zein CO, Unalp A, Colvin R, Liu YC, McCullough AJ, Nonalcoholic Steatohepatitis Clinical Research Network (2011) Smoking and severity of hepatic fibrosis in nonalcoholic fatty liver disease. J Hepatol 54:753-759

127. Tsochatzis EA, Papatheodoridis GV (2010) Smoking is associated with histological severity in nonalcoholic steatohepatitis. Hepatology $52: 1522-1523$ 
128. Argo CK, Northup PG, Al-Osaimi AM et al (2009) Systematic review of risk factors for fibrosis progression in non-alcoholic steatohepatitis. J Hepatol 51:371-379

129. Chiang DJ, Pritchard MT, Nagy LE (2011) Obesity, diabetes mellitus, and liver fibrosis. Am J Physiol Gastrointest Liver Physiol 300:G697-G702

130. Musso G, Gambino R, Cassader M (2011) Need for a threefocused approach to nonalcoholic fatty liver disease. Hepatology 53:1773

131. Preiss D, Khunti K, Sattar N (2011) Combined cardiovascular and diabetes risk assessment in primary care. Diabet Med 28: $19-22$

132. Silbernagel G, Grammer TB, Winkelmann BR, Boehm BO, März W (2011) Glycated hemoglobin predicts all-cause, cardiovascular, and cancer mortality in people without a history of diabetes undergoing coronary angiography. Diabetes Care. doi: $10.2337 / \mathrm{dc} 10-2010$

133. Abdul-Ghani MA, Williams K, DeFronzo RA, Stern M (2007) What is the best predictor of future type 2 diabetes? Diabetes Care 30:1544-1548

134. Musso G, Gambino R, Biroli G et al (2005) Hypoadiponectinemia predicts the severity of hepatic fibrosis and pancreatic beta-cell dysfunction in nondiabetic nonobese patients with nonalcoholic steatohepatitis. Am J Gastroenterol 100:2438-2446

135. Reddy KJ, Singh M, Bangit JR, Batsell RR (2010) The role of insulin resistance in the pathogenesis of atherosclerotic cardiovascular disease: an updated review. J Cardiovasc Med 11:633-647

136. Calori G, Lattuada G, Ragogna F et al (2011) Fatty liver index (FLI) and mortality: the Cremona study at the 15th year of follow up. Hepatology. doi:10.1002/hep.24356

137. Muniyappa R, Lee S, Chen H, Quon MJ (2008) Current approaches for assessing insulin sensitivity and resistance in vivo: advantages, limitations, and appropriate usage. Am J Physiol Endocrinol Metab 294:E15-E26

138. Devaraj S, Valleggi S, Siegel D, Jialal I (2010) Role of C-reactive protein in contributing to increased cardiovascular risk in metabolic syndrome. Curr Atheroscler Rep 12:110-118

139. Musso G, Gambino R, Cassader M, Pagano G (2010) A metaanalysis of randomized trials for the treatment of nonalcoholic fatty liver disease. Hepatology 52:79-104

140. Gambino R, Musso G, Cassader M (2011) Redox balance in the pathogenesis of nonalcoholic fatty liver disease: mechanisms and therapeutic opportunities. Antioxid Redox Signal 15:1325-1365 